Supporting Information (SI) for :

\title{
Measure-Specific Effectiveness of Air Pollution Control on China's Atmospheric Mercury Concentration and Deposition during 2013-2017
}

Kaiyun Liu, ${ }^{\dagger}$ Qingru Wu, ${ }^{\dagger}$ Long Wang,,${ }^{\dagger}$ Shuxiao Wang, ${ }^{*}, \dagger$, Tonghao Liu,,$\|$ Dian Ding,,${ }^{\dagger}$ Yi Tang,,${ }^{\dagger}$ Guoliang Li, ${ }^{\dagger}$

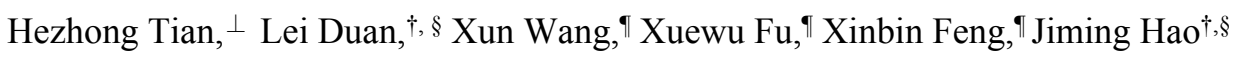

$\dagger$ State Key Joint Laboratory of Environment Simulation and Pollution Control, School of Environment, Tsinghua University, Beijing 100084, China

$¥$ School of Environment and Energy, South China University of Technology, Guangzhou 510006, China

\$State Environmental Protection Key Laboratory of Sources and Control of Air Pollution Complex, Beijing 100084, China

" China National Environmental Monitoring Centre, Beijing 100012, China

$\perp$ State Key Joint Laboratory of Environmental Simulation \& Pollution Control, School of Environment, Beijing Normal University, Beijing 100875, China

I State Key Laboratory of Environmental Geochemistry, Institute of Geochemistry, Chinese Academy of Sciences, Guiyang 550081, China

*Corresponding author. Tel.: +86 10 62771466; fax: +86 1062773597.

E-mail address: shxwang@tsinghua.edu.cn (S. Wang).

32 pages (including cover page)

12 Tables (S1, S2, S3, S4, S5, S6, S7, S8, S9, S10, S11, S12)

15 Figures (S1, S2, S3, S4, S5, S6, S7, S8, S9, S10, S11, S12, S13, S14, S15) 


\section{Methods for Mercury Emission Estimation}

In the CAMER model, mercury emission sources were divided into 29 categories (Table S1), including 13 categories of point sources and 16 categories of area sources. The key parameters in the model were activity levels (energy and material consumption, industrial products, etc.), mercury concentration in fuel/raw materials, and APCD related parameters (removal efficiencies, installation rates, and mercury speciation). During 20132017, the changing parameter values were activity levels, APCDs' types, and installation rates, while the mercury concentrations in fuel/raw materials, removal efficiencies and mercury speciation of one certain type of APCD in each province are assumed invariant. Based on the dominance of the $\mathrm{Hg}$ emission sources and the availability of essential data, all the categories were ranked into three tiers and methods for them were as follows:

(1) Tier 1 used the data for intentional use of mercury from the Report for National Mercury Investigation of China, including chlor-alkali production, caustic soda production, thermometer production, fluorescent lamp, sphygmomanometer production and battery production; ${ }^{1}$

(2) Tier 2 used the deterministic emission factor model with one single emission factor for the calculation of mercury emission from each sector (Table S2), including stationary oil combustion, mobile oil combustion, biomass fuel combustion, municipal solid waste incineration, hazardous wastes incineration, cremation, Al smelting, Hg smelting, large-scale gold production, artisanal and small-scale gold mining and oil refining;

(3) Tier 3 adopted the technology-based probabilistic emission factor model with provincial mercury concentration in fuel/raw material (Table S3-S4), APCD's types or provincial installation rates, including coalfired power plant, industrial coal-fired boiler, heating supply, residential coal combustion, other coal combustion, $\mathrm{Pb}$ smelting, $\mathrm{Zn}$ smelting, $\mathrm{Cu}$ smelting, cement clinker production, iron and steel production, coking and coal gasification. 
Table S1. Categories of mercury emission sources in China and technology breakdown

\begin{tabular}{|c|c|c|c|c|}
\hline Category & Source type & Tier & Ref. of Activity levels & $\begin{array}{l}\text { Ref. of APCDs' types and provincial installation } \\
\text { rates }\end{array}$ \\
\hline Coal-fired power plant & Point source & 3 & $\begin{array}{l}\text { Chinese statistics of electric power industry } \\
(2013-2017)^{2} \text { and Chinese environmental } \\
\text { statistics }(2013-2017)^{3}\end{array}$ & $\begin{array}{l}\text { Chinese statistics of electric power industry } \\
(2013-2017)^{2} \text { and Chinese environmental } \\
\text { statistics }(2013-2017)^{3}\end{array}$ \\
\hline $\begin{array}{l}\text { Industrial coal-fired } \\
\text { boiler }\end{array}$ & Area source & 3 & $\begin{array}{l}\text { China Energy Statistical Yearbook (2014-2017) } \\
{ }^{4} \text {, provincial statistical yearbooks (2018) and } \\
\text { the Chinese environmental statistics (2013- } \\
2017)^{3}\end{array}$ & $\begin{array}{l}\text { Chinese environmental statistics }(2013-2017)^{3} \text {, } \\
\text { Wu et al. }(2016)^{5} \text { and the management system } \\
\text { of pollutant emission permit } \\
\text { (http://permit.mee.gov.cn//) }\end{array}$ \\
\hline Heating supply & Area source & 3 & $\begin{array}{l}\text { China Energy Statistical Yearbook (2014-2017) } \\
{ }^{4} \text {, provincial statistical yearbooks (2018) and } \\
\text { the Chinese environmental statistics (2013- } \\
2017)^{3}\end{array}$ & $\begin{array}{l}\text { Chinese environmental statistics }(2013-2017)^{3} \text {, } \\
\text { Wu et al. }(2016)^{5} \text { and the management system } \\
\text { of pollutant emission permit } \\
\text { (http://permit.mee.gov.cn/ })^{6}\end{array}$ \\
\hline $\begin{array}{l}\text { Residential coal } \\
\text { combustion }\end{array}$ & Area source & 3 & $\begin{array}{l}\text { China Energy Statistical Yearbook (2014-2017) } \\
{ }^{4} \text {, provincial statistical yearbooks (2018) and } \\
\text { the Chinese environmental statistics (2013- } \\
2017)^{3}\end{array}$ & - \\
\hline Other coal combustion & Area source & 3 & $\begin{array}{l}\text { China Energy Statistical Yearbook (2014-2017) } \\
{ }^{4} \text {, provincial statistical yearbooks (2018) and } \\
\text { the Chinese environmental statistics (2013- } \\
2017)^{3}\end{array}$ & $\begin{array}{l}\text { Chinese environmental statistics }(2013-2017)^{3} \\
\text { and the management system of pollutant } \\
\text { emission permit (http://permit.mee.gov.cn/) }{ }^{6}\end{array}$ \\
\hline Mobile oil combustion & Area source & 2 & China Statistical Yearbook (2014-2018) ${ }^{7}$ & - \\
\hline $\begin{array}{l}\text { Stationary oil } \\
\text { combustion }\end{array}$ & Area source & 2 & China Statistical Yearbook (2014-2018) ${ }^{7}$ & - \\
\hline $\begin{array}{l}\text { Biomass fuel } \\
\text { combustion }\end{array}$ & Area source & 2 & China Statistical Yearbook (2014-2018) ${ }^{7}$ & - \\
\hline $\begin{array}{l}\text { Municipal solid waste } \\
\text { incineration }\end{array}$ & Point source & 2 & $\begin{array}{l}\text { China Energy Statistical Yearbook (2014-2017) } \\
4\end{array}$ & $\begin{array}{l}\text { Chinese environmental statistics (2013-2017) } \\
\text { and the management system of pollutant } \\
\text { emission permit ( } \underline{\text { http://permit.mee.gov.cn/) }}{ }^{6}\end{array}$ \\
\hline $\begin{array}{l}\text { Hazardous wastes } \\
\text { incineration }\end{array}$ & Point source & 2 & $\begin{array}{l}\text { China Energy Statistical Yearbook (2014-2017) } \\
4\end{array}$ & $\begin{array}{l}\text { Chinese environmental statistics }(2013-2017)^{3} \\
\text { and the management system of pollutant } \\
\text { emission permit (http://permit.mee.gov.cn/) }{ }^{6}\end{array}$ \\
\hline
\end{tabular}




\begin{tabular}{|c|c|c|c|c|}
\hline Cremation & Area source & 2 & China Statistical Yearbook $(2014-2018)^{7}$ & - \\
\hline $\mathrm{Pb}$ smelting & Point source & 3 & Chinese environmental statistics $(2013-2017)^{3}$ & $\begin{array}{l}\text { Chinese environmental statistics (2013-2017) } \\
\text { and the management system of pollutant } \\
\text { emission permit (http://permit.mee.gov.cn/) }{ }^{6}\end{array}$ \\
\hline Zn smelting & Point source & 3 & Chinese environmental statistics $(2013-2017)^{3}$ & $\begin{array}{l}\text { Chinese environmental statistics (2013-2017) } \\
\text { and the management system of pollutant } \\
\text { emission permit (http://permit.mee.gov.cn/) }{ }^{6}\end{array}$ \\
\hline $\mathrm{Cu}$ smelting & Point source & 3 & Chinese environmental statistics $(2013-2017)^{3}$ & $\begin{array}{l}\text { Chinese environmental statistics (2013-2017) } \\
\text { and the management system of pollutant } \\
\text { emission permit (http://permit.mee.gov.cn/) }{ }^{6}\end{array}$ \\
\hline Al smelting & Point source & 2 & Chinese environmental statistics $(2013-2017)^{3}$ & $\begin{array}{l}\text { Chinese environmental statistics (2013-2017) } \\
\text { and the management system of pollutant } \\
\text { emission permit (http://permit.mee.gov.cn/) }{ }^{6}\end{array}$ \\
\hline Hg smelting & Point source & 2 & Chinese environmental statistics $(2013-2017)^{3}$ & $\begin{array}{l}\text { Chinese environmental statistics (2013-2017) } \\
\text { and the management system of pollutant } \\
\text { emission permit (http://permit.mee.gov.cn/) }{ }^{6}\end{array}$ \\
\hline $\begin{array}{l}\text { Large-scale gold } \\
\text { production }\end{array}$ & Point source & 2 & Chinese environmental statistics $(2013-2017)^{3}$ & - \\
\hline $\begin{array}{l}\text { Artisanal and small- } \\
\text { scale gold smelting }\end{array}$ & Area source & 2 & Predicted based on Wu et al., (2016) ${ }^{5}$ & 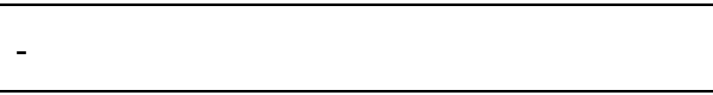 \\
\hline $\begin{array}{l}\text { Cement clinker } \\
\text { production }\end{array}$ & Point source & 3 & Chinese environmental statistics $(2013-2017)^{3}$ & $\begin{array}{l}\text { Chinese environmental statistics (2013-2017) } \\
\text { and the management system of pollutant } \\
\text { emission permit (http://permit.mee.gov.cn/) }{ }^{6}\end{array}$ \\
\hline $\begin{array}{l}\text { Iron and steel } \\
\text { production }\end{array}$ & Point source & 3 & Chinese environmental statistics $(2013-2017)^{3}$ & $\begin{array}{l}\text { Chinese environmental statistics (2013-2017) } \\
\text { and the management system of pollutant } \\
\text { emission permit (http://permit.mee.gov.cn/ })^{6}\end{array}$ \\
\hline Coking & Point source & 3 & Chinese environmental statistics $(2013-2017)^{3}$ & $\begin{array}{l}\text { Chinese environmental statistics (2013-2017) } \\
\text { and the management system of pollutant } \\
\text { emission permit (http://permit.mee.gov.cn/) }{ }^{6}\end{array}$ \\
\hline Coal gasification & Area source & 3 & China Statistical Yearbook (2014-2018) ${ }^{7}$ & $\begin{array}{l}\text { Chinese environmental statistics (2013-2017) } \\
\text { and the management system of pollutant } \\
\text { emission permit (http://permit.mee.gov.cn/) }{ }^{6}\end{array}$ \\
\hline
\end{tabular}




\begin{tabular}{|c|c|c|c|c|}
\hline Oil refining & Area source & 2 & 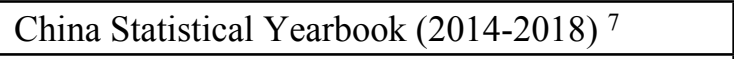 & - \\
\hline $\begin{array}{l}\text { Chlor-alkali } \\
\text { production }\end{array}$ & Point source & 1 & $\begin{array}{l}\text { Chinese environmental statistics }(2013-2017)^{3} \\
\text { and the Report of National Mercury } \\
\text { Investigation of China }{ }^{1}\end{array}$ & - \\
\hline $\begin{array}{l}\text { Caustic soda } \\
\text { production }\end{array}$ & Area source & 1 & $\begin{array}{l}\text { China Statistical Yearbook }(2014-2018)^{7} \text { and } \\
\text { the Report of National Mercury Investigation of } \\
\text { China }{ }^{1}\end{array}$ & - \\
\hline $\begin{array}{l}\text { Thermometer } \\
\text { production }\end{array}$ & Area source & 1 & $\begin{array}{l}\text { China Statistical Yearbook (2014-2018) }{ }^{7} \text { and } \\
\text { the Report of National Mercury Investigation of } \\
\text { China }{ }^{1}\end{array}$ & - \\
\hline Fluorescent lamp & Area source & 1 & $\begin{array}{l}\text { China Statistical Yearbook }(2014-2018)^{7} \text { and } \\
\text { the Report of National Mercury Investigation of } \\
\text { China }{ }^{1}\end{array}$ & - \\
\hline $\begin{array}{l}\text { Sphygmomanometer } \\
\text { production }\end{array}$ & Area source & 1 & $\begin{array}{l}\text { China Statistical Yearbook }(2014-2018)^{7} \text { and } \\
\text { the Report of National Mercury Investigation of } \\
\text { China }{ }^{1}\end{array}$ & - \\
\hline Battery production & Area source & 1 & $\begin{array}{l}\text { China Statistical Yearbook }(2014-2018)^{7} \text { and } \\
\text { the Report of National Mercury Investigation of } \\
\text { China }{ }^{1}\end{array}$ & - \\
\hline
\end{tabular}




\section{Changes of Activity Levels during 2013-2017}

In this study, point sources included 1823 coal-fired power plants, 1980 cement clinkers, 305 Zn smelting plants, $252 \mathrm{~Pb}$ smelting plants, $152 \mathrm{Cu}$ smelting plants, $220 \mathrm{Al}$ smelting plants, $10 \mathrm{Hg}$ smelting plants, 42 largescale gold smelting plants, 610 waste incineration plants, 2350 iron and steel plants, 550 coking plants and 180 chlor-alkali production plants. As shown in Table S1, the activity level of each coal-fired power plant was obtained from Chinese statistics of electric power industry database (2013-2017) and Chinese environmental statistics (2013-2017). ${ }^{2,3}$ For the rest point sources, activity levels were mainly from the Chinese environmental statistics (2013-2017). ${ }^{3}$ The bottom-up provincial activity levels are verified by the corresponding data in China Energy Statistical Yearbook (2014-2017) and China Statistical Yearbook (2014-2018). ${ }^{4} 7$ As shown in Figure S1, power plants are concentrated in or near the coastal provinces (Shandong, Jiangsu, Zhejiang) and coalproducing provinces (Inner Mongolia, Guizhou, Shanxi); non-ferrous metals smelting plants are dense in Hunan, Yunnan and Henan; waste incineration plants are concentrated in Jiangsu, Zhejiang and Guangdong; iron and steel smelting plants are dense in Hebei, Shanxi and Sichuan.

As to the 16 categories of area sources in Table S1, the provincial activity levels were mainly derived from China Energy Statistical Yearbook (2014-2017) and China Statistical Yearbook (2014-2018). ${ }^{4,7}$ It's worth noting that the provincial activity levels of industrial coal-fired boiler, heating supply, residential coal combustion, other coal combustion in 2017 were predicted based on the provincial statistical yearbooks (2018) of Beijing, Shanxi, Jiangsu, Zhejiang, Fujian, Guangdong, Guangxi, Hainan, Chongqing, Guizhou, Shaanxi, and Xinjiang, as well as Energy Statistical Yearbook (2017) and the Chinese environmental statistics (2017). ${ }^{3,4}$ 


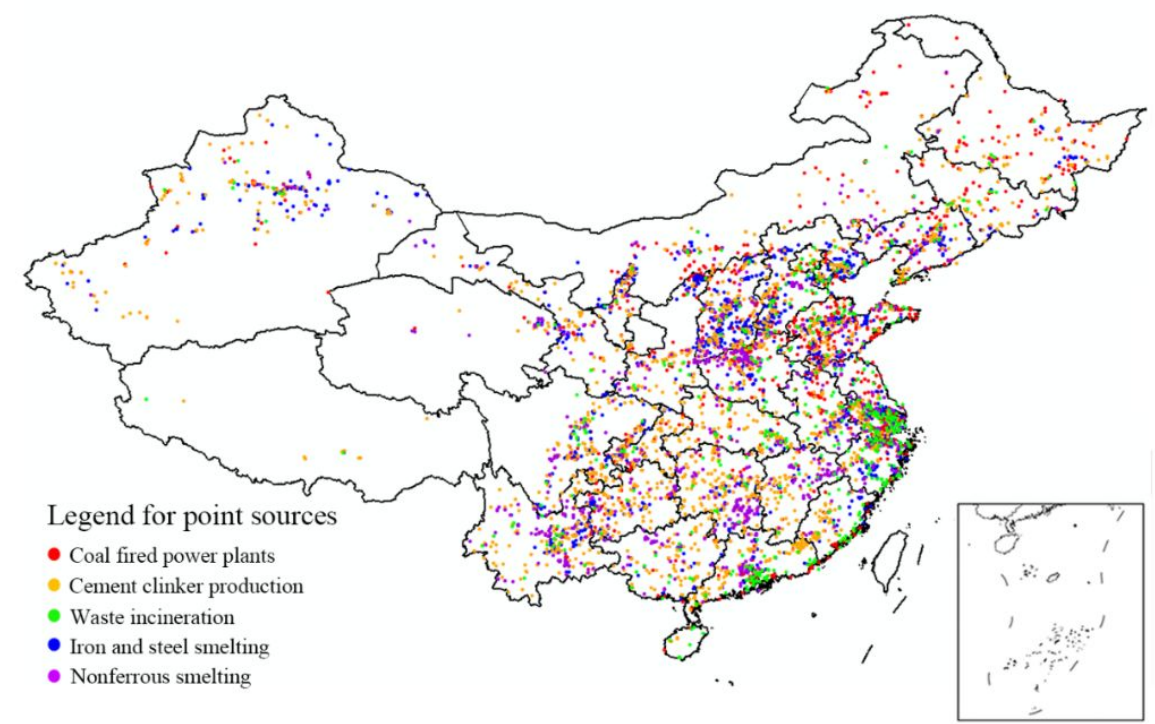

Figure S1. Geographical distribution of point sources in 2017

Figure S2 shows the activity level trends of main sources during 2013-2017. It should be noted that the activity level of a certain non-ferrous metal smelting plant is the amount of consumed metal concentrate based on the Chinese environmental statistics (2013-2017). ${ }^{3}$ If lack of the amount of consumed metal concentrate, it would be converted from the amount of metal product based on the grade of the concentrate $(50,63$ and 22 for $\mathrm{Zn}, \mathrm{Pb}$ and $\mathrm{Cu}$ respectively) and the metal recovery rate (95, 95 and $97 \mathrm{for} \mathrm{Zn}, \mathrm{Pb}$ and $\mathrm{Cu}$ respectively). Similarly, the activity level of a certain cement clinker plant was limestone based on the Chinese environmental statistics (2013-2017). ${ }^{3}$ If lack of the amount of consumed limestion, it would be converted from the amount of clinker based on the limestone/clinker ratio (1.5). Previous studies found that the mercury emissions from coal combustion, building material production and metal smelters accounted for over $85 \%$ of China's total anthropogenic mercury emissions. ${ }^{5,8}$ The activity level trends of these sectors are given in Figure S2. During 2013-2017, the amount of coal consumption decreased 3\%, the production of cement clinker increased by $3 \%$, the production of iron and steel increased by $2 \%$, the production of $\mathrm{Pb}$ smelting increased by $6 \%$, the production of $\mathrm{Zn}$ smelting increased by $17 \%$. 


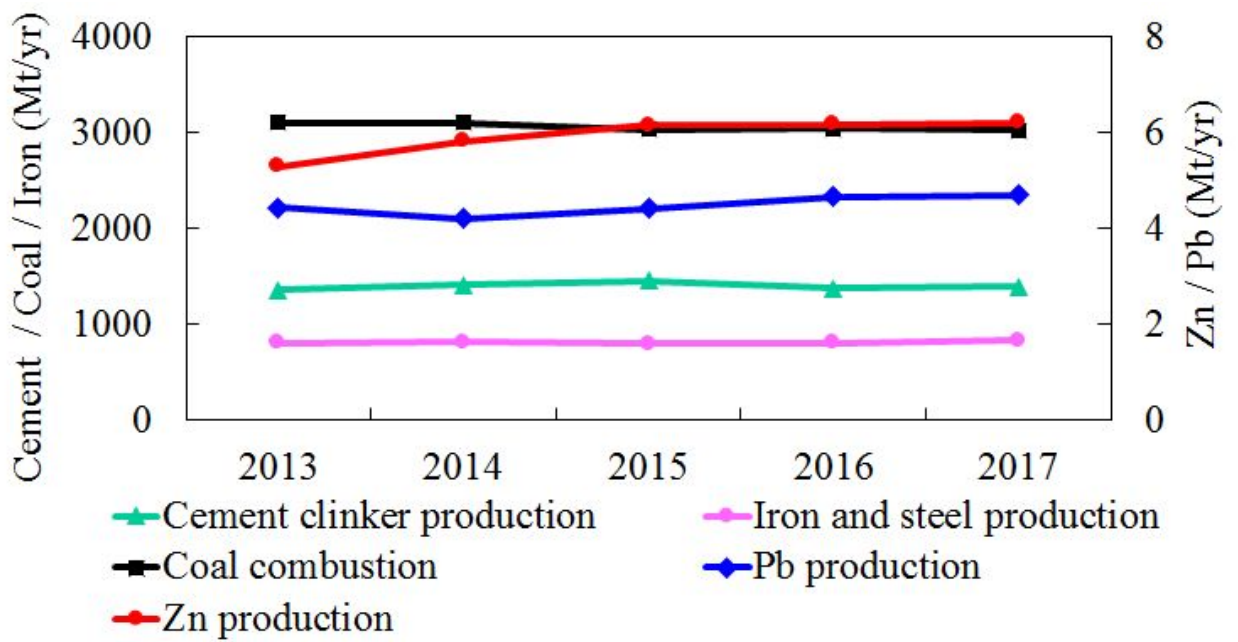

Figure S2. Activity level trends of main sources during 2013-2017

\section{Mercury emission factors and mercury speciation for the mercury emission sources in Tier2}

Emission factors and mercury speciation for the mercury emission sources in Tier 2 are listed in Table S2.

Table S2. Emission factors and mercury speciation for mercury emission sources in Tier2

\begin{tabular}{|l|l|l|l|l|l|}
\hline \multirow{2}{*}{ Emission source } & \multirow{2}{*}{ Emission factor } & Unit & \multicolumn{3}{l|}{ Mercury speciation (\%) } \\
\cline { 3 - 6 } & & & GEM & GOM & PBM \\
\hline Stationary oil combustion (diesel) & 0.0065 & $\mathrm{~g} / \mathrm{t}$ & 50 & 40 & 10 \\
\hline Mobile oil combustion (diesel) & 0.0065 & $\mathrm{~g} / \mathrm{t}$ & 50 & 40 & 10 \\
\hline Mobile oil combustion (gasoline) & 0.00362 & $\mathrm{~g} / \mathrm{t}$ & 50 & 40 & 10 \\
\hline Biomass fuel combustion & 0.01 & $\mathrm{~g} / \mathrm{t}$ & 74 & 5 & 21 \\
\hline Municipal solid waste incineration & 0.5 & $\mathrm{~g} / \mathrm{t}$ & 96 & 0 & 4 \\
\hline Hazardous wastes incineration & 0.5 & $\mathrm{~g} / \mathrm{t}$ & 96 & 0 & 4 \\
\hline Cremation & 1.0 & $\mathrm{~g} / \mathrm{corpse}$ & 96 & 0 & 4 \\
\hline Al smelting & 0.265 & $\mathrm{~g} / \mathrm{t}$ & 85 & 15 & 5 \\
\hline Hg smelting & 2.0 & $\mathrm{~g} / \mathrm{kg}$ & 85 & 15 & 5 \\
\hline Large-scale gold production & 0.026 & $\mathrm{~g} / \mathrm{g}$ & 85 & 15 & 5 \\
\hline Artisanal and small-scale gold mining & 2.0 & $\mathrm{~g} / \mathrm{g}$ & 85 & 15 & 5 \\
\hline Oil refining & 0.002075 & $\mathrm{~g} / \mathrm{t}$ & 50 & 40 & 10 \\
\hline
\end{tabular}




\section{Provincial Mercury Concentration in Fuels/Raw Materials}

Mercury concentration raw coal samples, zinc concentrate samples, lead concentrate samples, copper concentrate samples, limestone samples were collected from our previous studies (Table S3), which were all found to fit the lognormal distribution. ${ }^{13-15}$ With the inter-provincial transport matrices for coal and non-ferrous metal concentrates (Wu et al., 2012), we converted mercury concentrations in mined coal and metal concentrates into that in consumed coal and metal concentrates. ${ }^{14,} 16$ The provincial default values were replaced by the national average values. 
Table S3. Provincial Hg contents in produced and consumed coal, limestone and iron ore of China, unit: $g / t$

\begin{tabular}{|c|c|c|c|c|c|c|c|c|c|c|c|c|}
\hline \multirow{2}{*}{ Province } & \multicolumn{3}{|c|}{ Produced coal } & \multirow{2}{*}{$\begin{array}{c}\text { Consumed } \\
\text { coal } \\
\end{array}$} & \multicolumn{3}{|c|}{ Mined Limestone } & \multirow{2}{*}{$\begin{array}{l}\text { Consumed } \\
\text { limestone }\end{array}$} & \multicolumn{3}{|c|}{ Mined iron ore } & \multirow{2}{*}{$\begin{array}{c}\text { Consumed } \\
\text { iron ore }\end{array}$} \\
\hline & $\mathrm{AM}$ & $\mathrm{SD}$ & NS & & AM & $\mathrm{SD}$ & NS & & $\mathrm{AM}$ & $\mathrm{SD}$ & $\mathrm{NS}$ & \\
\hline Beijing & - & - & - & 0.18 & - & - & - & 0.021 & - & - & - & 0.038 \\
\hline Tianjin & - & - & - & 0.14 & - & - & - & 0.021 & 0.041 & 0.008 & 3 & 0.041 \\
\hline Hebei & 0.17 & 0.12 & 24 & 0.18 & 0.548 & 0.727 & 17 & 0.548 & 0.037 & 0.04 & 79 & 0.037 \\
\hline Shanxi & 0.15 & 0.15 & 88 & 0.15 & - & - & - & 0.021 & 0.03 & 0.028 & 25 & 0.03 \\
\hline Inner Mongolia & 0.18 & 0.25 & 46 & 0.18 & - & - & - & 0.021 & - & - & - & 0.038 \\
\hline Liaoning & 0.14 & 0.14 & 19 & 0.16 & - & - & - & 0.021 & - & - & - & 0.038 \\
\hline Jilin & 0.07 & 0.01 & 5 & 0.16 & - & - & - & 0.021 & - & - & - & 0.038 \\
\hline Heilongjiang & 0.05 & 0.03 & 20 & 0.11 & - & - & - & 0.021 & - & - & - & 0.038 \\
\hline Shanghai & - & - & - & 0.16 & - & - & - & 0.021 & 0.044 & 0.05 & 4 & 0.044 \\
\hline Jiangsu & 0.27 & 0.19 & 11 & 0.2 & 0.109 & 0.114 & 17 & 0.109 & - & - & - & 0.038 \\
\hline Zhejiang & - & - & - & 0.17 & 0.052 & 0.035 & 22 & 0.052 & - & - & - & 0.038 \\
\hline Anhui & 0.2 & 0.15 & 20 & 0.2 & 0.011 & 0.004 & 16 & 0.011 & 0.021 & 0.012 & 14 & 0.021 \\
\hline Fujian & 0.07 & 0.06 & 3 & 0.17 & 0.011 & 0.005 & 4 & 0.011 & 0.019 & 0.011 & 15 & 0.019 \\
\hline Jiangxi & 0.27 & 0.17 & 7 & 0.21 & 0.015 & 0.02 & 9 & 0.015 & 0.057 & 0.088 & 18 & 0.057 \\
\hline Shandong & 0.16 & 0.09 & 33 & 0.16 & 0.499 & 0.626 & 21 & 0.499 & 0.125 & 0.073 & 20 & 0.125 \\
\hline Henan & 0.17 & 0.11 & 37 & 0.15 & 0.747 & 0.623 & 12 & 0.747 & 0.032 & 0.029 & 18 & 0.032 \\
\hline Hubei & 0.16 & 0.07 & 3 & 0.21 & 0.1 & 0.076 & 6 & 0.1 & - & - & - & 0.038 \\
\hline Hunan & 0.14 & 0.1 & 10 & 0.21 & 0.1 & 0.076 & 3 & 0.1 & 0.033 & 0.034 & 77 & 0.033 \\
\hline Guangdong & 0.06 & 0.04 & 2 & 0.15 & 0.048 & 0.026 & 15 & 0.048 & - & - & - & 0.038 \\
\hline Guangxi & 0.35 & 0.27 & 5 & 0.17 & 0.008 & 0.002 & 4 & 0.008 & - & - & - & 0.038 \\
\hline Hainan & - & - & - & 0.1 & - & - & - & 0.021 & - & - & - & 0.038 \\
\hline Chongqing & 0.41 & 0.2 & 12 & 0.37 & - & - & - & 0.021 & - & - & - & 0.038 \\
\hline Sichuan & 0.33 & 0.14 & 15 & 0.3 & 0.017 & 0.011 & 13 & 0.017 & 0.027 & 0.044 & 7 & 0.027 \\
\hline Guizhou & 0.21 & 0.34 & 46 & 0.21 & - & - & - & 0.021 & - & - & - & 0.038 \\
\hline Yunnan & 0.13 & 0.1 & 17 & 0.13 & 0.018 & 0.007 & 4 & 0.018 & 0.1 & 0.007 & 6 & 0.1 \\
\hline Xizang & - & - & - & 0.17 & - & - & - & 0.021 & - & - & - & 0.038 \\
\hline Shaanxi & 0.25 & 0.28 & 28 & 0.25 & 0.012 & 0.01 & 4 & 0.012 & - & - & - & 0.038 \\
\hline Gansu & 0.18 & 0.11 & 7 & 0.13 & - & - & - & 0.021 & 0.107 & 0.003 & 3 & 0.107 \\
\hline Qinghai & 0.05 & - & 1 & 0.17 & - & - & - & 0.021 & - & - & - & 0.038 \\
\hline Ningxia & 0.21 & 0.01 & 4 & 0.19 & - & - & - & 0.021 & - & - & - & 0.038 \\
\hline Xinjiang & 0.03 & 0.01 & 18 & 0.05 & - & - & - & 0.021 & 0.006 & 0.004 & 17 & 0.006 \\
\hline Imported & 12 & 0.17 & 0.1 & 0.17 & - & - & - & 0.021 & - & - & - & 0.038 \\
\hline National & 494 & 0.16 & 0.19 & 0.17 & 0.201 & 0.435 & 167 & 0.201 & 0.038 & 0.048 & 306 & 0.038 \\
\hline
\end{tabular}

Note: AM - arithmetic mean, SD - standard deviation, NS - number of samples. 
Table S4. Provincial Hg content in mined and consumed $\mathrm{Zn} / \mathrm{Pb} / \mathrm{Cu}$ concentrate of $\mathrm{China}$, unit: $\mathrm{g} / \mathrm{t}$

\begin{tabular}{|c|c|c|c|c|c|c|c|c|c|c|c|c|}
\hline \multirow{2}{*}{ Province } & \multicolumn{3}{|c|}{ Mined Zn concentrate } & \multirow{2}{*}{$\begin{array}{c}\text { Consumed } \\
\text { Zn concentrate }\end{array}$} & \multicolumn{3}{|c|}{ Mined $\mathrm{Pb}$ concentrate } & \multirow{2}{*}{$\begin{array}{c}\text { Consumed } \\
\mathrm{Pb} \text { concentrate }\end{array}$} & \multicolumn{3}{|c|}{ Mined $\mathrm{Cu}$ concentrate } & \multirow{2}{*}{$\begin{array}{c}\text { Consumed } \\
\text { Cu concentrate }\end{array}$} \\
\hline & $\mathrm{AM}$ & $\mathrm{SD}$ & $\mathrm{NS}$ & & AM & $\mathrm{SD}$ & $\mathrm{NS}$ & & $\mathrm{AM}$ & $\mathrm{SD}$ & $\mathrm{NS}$ & \\
\hline Beijing & - & - & - & 77.5 & - & - & - & 31.4 & - & - & - & 5.8 \\
\hline Tianjin & - & - & - & 77.5 & - & - & - & 31.4 & - & - & - & 5.8 \\
\hline Hebei & - & - & - & 77.5 & - & - & - & 31.4 & - & - & - & 2.42 \\
\hline Shanxi & - & - & - & 77.5 & 52.17 & 0.41 & 3 & 52.17 & 0.19 & 0.15 & 9 & 5.08 \\
\hline Inner Mongolia & 6.19 & 10.69 & 45 & 13.45 & 54.47 & 25.37 & 9 & 62.21 & 1.9 & 0.52 & 4 & 5.56 \\
\hline Liaoning & - & - & - & 13.77 & 69.33 & 29.92 & 6 & 61.04 & - & - & - & 5.48 \\
\hline Jilin & - & - & - & 77.5 & 58.67 & 22.72 & 3 & 31.4 & - & - & - & 5.8 \\
\hline Heilongjiang & - & - & - & 77.5 & 26.28 & 6.94 & 4 & 31.4 & - & - & - & 5.8 \\
\hline Shanghai & - & - & - & 77.5 & - & - & - & 31.4 & - & - & - & 5.8 \\
\hline Jiangsu & 25.5 & 13.69 & 4 & 77.5 & 33.86 & 32.71 & 10 & 31.4 & 0.06 & 0.01 & 3 & 0.73 \\
\hline Zhejiang & 1.53 & 1.63 & 9 & 0.88 & 33.31 & 44.68 & 9 & 31.4 & - & - & - & 4.31 \\
\hline Anhui & 4.1 & - & 1 & 2.65 & 25.34 & 45.78 & 4 & 9.64 & 0.72 & 0.96 & 12 & 3.44 \\
\hline Fujian & 1.42 & 2.89 & 44 & 0.54 & 18.89 & 21.76 & 11 & 12.63 & - & - & - & 6.36 \\
\hline Jiangxi & 4.54 & 4.12 & 35 & 1.47 & 19.52 & 0.77 & 2 & 19.28 & 8.14 & 15.47 & 31 & 5.93 \\
\hline Shandong & - & - & - & 77.5 & 4.99 & 1.16 & 2 & 31.4 & 1.6 & 0.52 & 10 & 7.06 \\
\hline Henan & 6.46 & 4.4 & 4 & 24.62 & 14.84 & 20.78 & 17 & 30.12 & - & - & - & 2.82 \\
\hline Hubei & - & - & - & 77.5 & 6.89 & 0.8 & 2 & 31.4 & 2.84 & 4.46 & 18 & 4.22 \\
\hline Hunan & 7.25 & 14.84 & 67 & 42.65 & 3.69 & 2.94 & 29 & 23.27 & - & - & - & 3.53 \\
\hline Guangdong & 266.31 & 199.9 & 27 & 35.06 & 54.44 & 49.02 & 10 & 35.17 & 0.05 & 0.01 & 3 & 5.8 \\
\hline Guangxi & 34.87 & 46.64 & 12 & 11.52 & 32.83 & 55.59 & 12 & 9.65 & 1.28 & 1.1 & 3 & 5.8 \\
\hline Hainan & - & - & - & 77.5 & - & - & - & 31.4 & - & - & - & 5.8 \\
\hline Chongqing & - & - & - & 77.5 & 115.06 & 7.02 & 3 & 31.4 & - & - & - & 5.8 \\
\hline Sichuan & 48.13 & 40.95 & 32 & 59.23 & 57.46 & 66.29 & 15 & 31.4 & 2.62 & 1.93 & 3 & 5.71 \\
\hline Guizhou & - & - & - & 3.47 & - & - & - & 31.4 & - & - & - & 5.8 \\
\hline Yunnan & 23.65 & 27.31 & 17 & 20.74 & 21.51 & 3.09 & 7 & 21.59 & 43.32 & 42.21 & 43 & 9.46 \\
\hline Xizang & 0.46 & 0.71 & 10 & 77.5 & 0.02 & 0.01 & 4 & 31.4 & - & - & - & 5.8 \\
\hline Shaanxi & 678.22 & 875.93 & 13 & 80.1 & 47.58 & 38.98 & 8 & 45.14 & - & - & - & 5.8 \\
\hline Gansu & 769.76 & 481.32 & 11 & 240.48 & 11.5 & 5.91 & 10 & 10.77 & 9.88 & 12.74 & 12 & 4.92 \\
\hline Qinghai & - & - & - & 20.02 & 1.1 & 1.16 & 10 & 0.6 & 1.78 & 0.05 & 2 & 5.8 \\
\hline Ningxia & - & - & - & 77.5 & - & - & - & 31.4 & - & - & - & 5.8 \\
\hline Xinjiang & 27.59 & 38.93 & 15 & 16.86 & - & - & - & 41.52 & 10.83 & 18.59 & 21 & 2.02 \\
\hline Imported & 32.04 & 81.98 & 35 & 77.5 & 3.54 & 2.38 & 8 & 31.4 & 1.93 & 2.71 & 33 & 5.8 \\
\hline National & 77.45 & 257.53 & 381 & 77.5 & 28 & 31.4 & 198 & 31.4 & 12.68 & 26.51 & 207 & 5.8 \\
\hline
\end{tabular}




\section{Changes of APCDs' Application Rates during 2013-2017 and Mercury Speciation in Exhausted Flue Gas}

As shown in Table S1, APCD type of each coal-fired power plant was obtained from database of Chinese statistics of electric power industry database (2013-2017) and Chinese environmental statistics (2013-2017). ${ }^{2,3}$ For the other point sources, APCD types were mainly from the Chinese environmental statistics (2013-2017) and the management system of pollutant emission permit (http://permit.mee.gov.cn/). ${ }^{3,6}$

The provincial installation rates of APCDs for 16 subcategories of area sources in 2013-2014 were obtained from Wu et al. (2016). ${ }^{5}$ The provincial installation rates of APCDs for area sources in 2015-2017 were mainly from the Chinese environmental statistics (2013-2017), meanwhile the provincial installation rates were verified by industrial association databases. ${ }^{3}$

From 2013 to 2017, the most remarkable change of APCD types and application rates is the $\mathrm{SO}_{2}$, NOx and $\mathrm{PM}_{2.5}$ control devices applied in coal-fired power plants and industrial coal-fired boilers. Figure $\mathbf{S} 3$ shows the provincial APCDs' constitution in 2013 and 2017 for coal-fired power plants. Figure S4 shows the provincial APCDs' constitution in 2013 and 2017 for industrial coal-fired boilers. Table S5 shows the national APCDs' application rates and mercury speciation in exhausted flue gas for main point sources.

Since 2013, a series of control polices for $\mathrm{SO}_{2}, \mathrm{NOx}$ and $\mathrm{PM}_{2.5}$ have been implemented. Both the APCD types and installation rates in main anthropogenic mercury emission sources changed significantly during 20132017. Different APCDs have different synergistic removal efficiencies. ${ }^{13}, 17$ Electrostatic precipitator (ESP), fabric filter (FF), advanced electrostatic fabric filter (ESP-FF), wet electrostatic precipitators (WESP) and low temperature electrostatic precipitator (LTESP), are applied as dust collectors, and the latter two can achieve higher performance in mercury removal. ${ }^{18}, 19$ The catalysts of selective catalytic reduction devices (SCR) can promote the oxidation of some of the GEM to GOM, which contributes to additional reduction of GOM in gypsum of wet flue gas desulfurization devices (WFGD). As shown in Figure S3, both APCDs for ultra-low emission - SCR+ESP-FF (LTESP) +WFGD and SCR+ESP+WFGD+WESP - have been increasingly retrofitted or built in coal-fired power plants to attain the stringent PM emission limits (GB13223-2011). ${ }^{16,20,21}$ SCR was commonly applied in pulverized coal boilers in 2017 , whereas its application rate was only $45 \%$ in $2013 .{ }^{22}$ The proportion of APCDs for ultra-low emission increased from $0 \%$ in 2013 to $65 \%$ in $2017 .{ }^{22}$ Field testing results 
show that ultra-low emission control is the most effective measure for flue gas mercury removal in coal-fired power plants. ${ }^{18,23-25}$

For industrial coal-fired boilers, cyclone (CYC) and wet scrubber (Wet) were the main dust removal devices in 2013, accounting for $68 \%$ of dust collectors (see Figure S4). The mercury removal efficencies of CYC and Wet are only $1 \%$ and $23 \%$, which are much lower than the mercury removal efficencies of ESP and FF. Since 2013, CYC and Wet have been gradually replaced by integrated dust removal device (IDRD), ESP and FF which could achieve much higher mercury emission removal efficencies. By the end of $2017,45 \%$ of the industrial coal-fired boilers installed IDRD and 55\% equipped with ESP/FF. The other one is that the appication rate of wet flue gas desulfurization (WFGD) increased from 5\% in 2013 to $45 \%$ in 2017.

For cement clinkers, the proportion of dry-process precalciner technology with dust recycling was about $92 \%$ in 2013. Since 2013, the newly built cement clinkers have adopted low nitrogen combustion technology, higheffective dust collectors and denitrification facilities to attain the stringent emission limits (GB4915-2013). ${ }^{26}$ The installation rate of SCR/SNCR in cement plants increased from 30\% in 2013 to $90 \%$ in 2017. However, due to the dry-process precalciner technology with dust recycling, the dust removed by ESP/FF is recycled as raw material, which leads to the invalidation of the synergistic mercury removal efficiency of ESP/FF. Field tests showed that the average mercury removal efficiency of SNCR+ESP/FF is only about $15 \% .{ }^{12,26,27}$

For $\mathrm{Zn}$ and $\mathrm{Pb}$ smelting plants, the combination of dust collector (DC), flue gas scrubber (FGS), electrostatic demister (ESD), acid plant with double contact and double absorption towers (APD) - DC+FGS+ESD+APD accounted for about $67 \%$ and $50 \%$ in $2013 .{ }^{5}$ Since 2013, those plants without APCDs have been gradually eliminated and flue gas desulfurization devices have come into popular. By the end of 2017, the proportions of $\mathrm{Zn}$ and $\mathrm{Pb}$ smelting plants equipped with FGDs have reached to $27 \%$ and $40 \%$.

During 2013-2017, APCDs for desulphurization and denitrogenation in iron and steel production were not generalized. ${ }^{15}$ Thereinto, the proportion of WFGD increased from $13 \%$ in 2013 to $21 \%$ in 2017 . Few iron and steel plants were installed with denitrogenation devices during 2013-2017. 


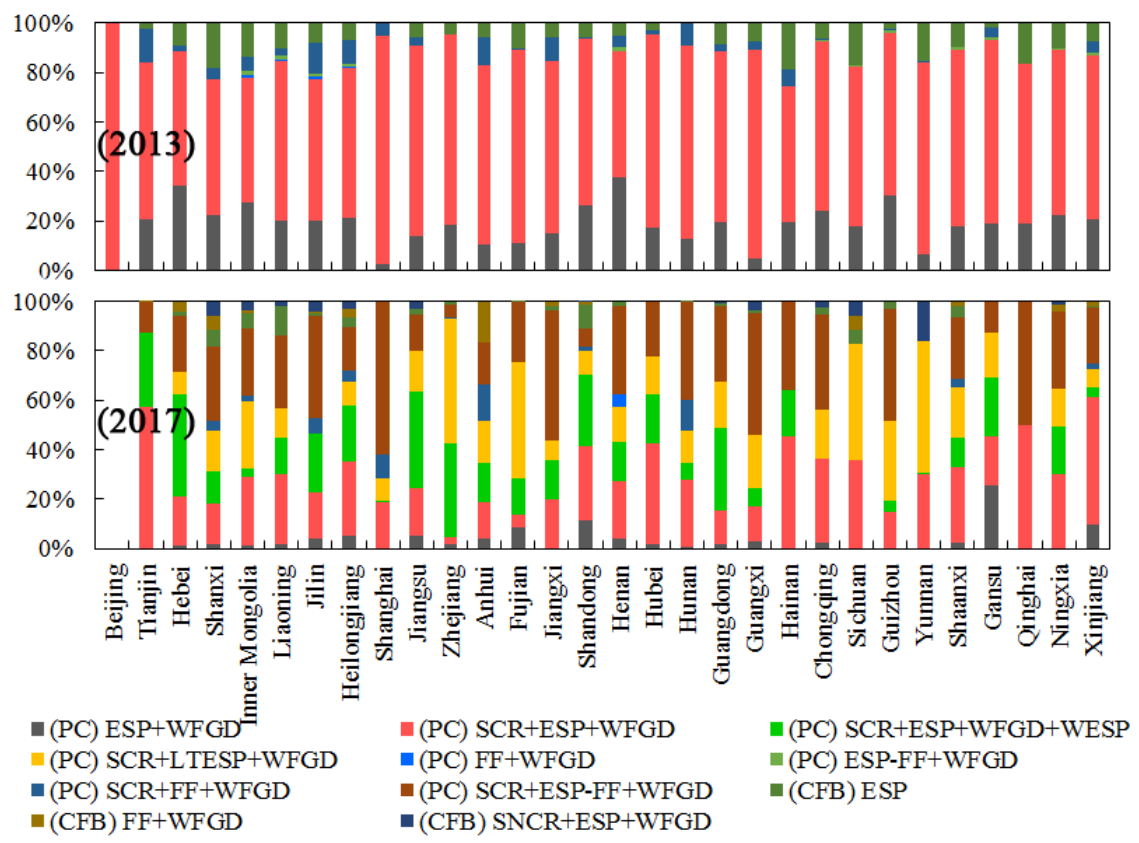

Figure S3. Provincial APCDs' constitution in 2013 (a) and 2017 (b) for coal-fired power plants. (PC - pulverized coal furnace; CFB - circulating fluidized bed; ESP - electrostatic precipitator; FF - fabric filter; WFGD - wet flue gas desulfurization; SCR - selective catalytic reduction; ESP-FF - electrostatic fabric filter; WESP - wet electrostatic precipitator, LTESP - low temperature electrostatic precipitator, SCR - selective catalytic reduction; SNCR - selective non-catalytic reduction.)

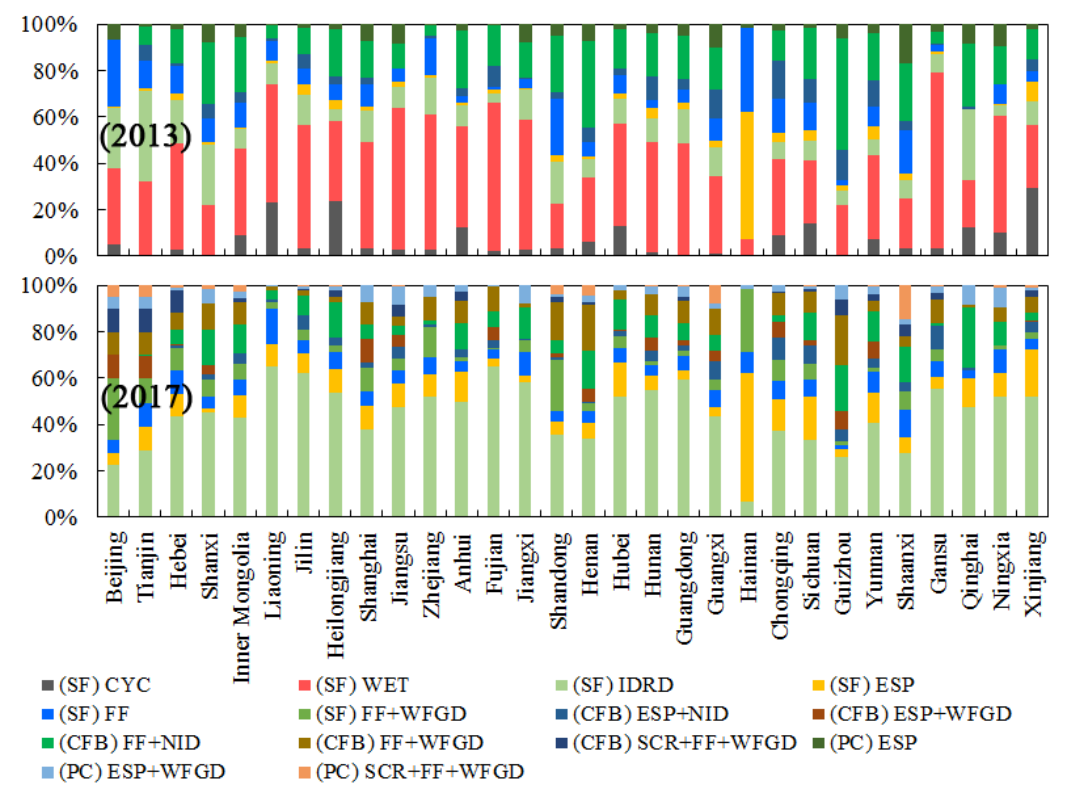

Figure S4. Provincial APCDs' constitution in 2013 (a) and 2017 (b) for industrial coal-fired boilers. (SF - stoker furnace; CYC - Cyclone, WET - wet scrubber, NID - novel integrated desulphurization; IDRD - integrated dust removal device) 
Table S5. National APCDs' application rates and mercury speciation in exhausted flue gas (\%)

\begin{tabular}{|c|c|c|c|c|c|c|}
\hline \multirow{2}{*}{ Emission source } & \multirow{2}{*}{ APCD type } & \multicolumn{2}{|c|}{ Installation rate } & \multicolumn{3}{|c|}{ Mercury speciation } \\
\hline & & 2013 & 2017 & GEM & GOM & PBM \\
\hline \multirow{14}{*}{ Coal-fired power plants ${ }^{16}$} & (PC) ESP+WFGD & 29 & 1 & 82 & 18 & 0 \\
\hline & (PC) FF+WFGD & 5 & 1 & 81 & 18 & 1 \\
\hline & (PC) ESP-FF+WFGD & 2 & 1 & 87 & 12 & 1 \\
\hline & (PC) SCR+ESP+WFGD & 40 & 23 & 78 & 22 & 0 \\
\hline & (PC) SCR+FF+WFGD & 3 & 5 & 35 & 62 & 3 \\
\hline & (PC) SCR+ESP+WFGD+WESP & 1 & 25 & 66 & 33 & 1 \\
\hline & (PC) SCR+LTESP+WFGD & 1 & 20 & 65 & 34 & 1 \\
\hline & (PC) SCR+ESP-FF+WFGD & 5 & 27 & 74 & 23 & 3 \\
\hline & (PC) SCR+ESP+SW-FGD & 1 & 1 & 78 & 22 & 0 \\
\hline & (PC) NID+ESP & 3 & 1 & 0 & 81 & 18 \\
\hline & (PC) ESP+CFB-FGD+FF & 1 & 1 & 67 & 33 & 0 \\
\hline & (CFB) ESP & 10 & 9 & 72 & 28 & 1 \\
\hline & (CFB) FF & 0 & 1 & 82 & 18 & 1 \\
\hline & (CFB) SNCR+ESP+WFGD & 0 & 1 & 51 & 48 & 1 \\
\hline \multirow{14}{*}{$\begin{array}{l}\text { Industrial coal-fired boilers, } \\
\text { heating supply, other coal } \\
\text { combustion }^{5,8}\end{array}$} & (SF) CYC & 18 & 5 & 38 & 38 & 24 \\
\hline & (SF) WET & 50 & 5 & 65 & 33 & 2 \\
\hline & (SF) IDRD & 12 & 55 & 49 & 48 & 3 \\
\hline & (SF) ESP & 3 & 3 & 58 & 41 & 1 \\
\hline & (SF) FF & 2 & 3 & 51 & 49 & 1 \\
\hline & (SF) FF+WFGD & 0 & 2 & 78 & 21 & 1 \\
\hline & (CFB) ESP+NID & 4 & 3 & 0 & 82 & 18 \\
\hline & (CFB) ESP+WFGD & 0 & 2 & 83 & 16 & 1 \\
\hline & (CFB) FF+NID & 7 & 5 & 0 & 82 & 18 \\
\hline & (CFB) FF+WFGD & 0 & 8 & 78 & 21 & 1 \\
\hline & (CFB) SCR+FF+WFGD & 0 & 1 & 37 & 61 & 2 \\
\hline & (PC) ESP & 4 & 4 & 58 & 41 & 1 \\
\hline & (PC) ESP+WFGD & 0 & 3 & 83 & 16 & 1 \\
\hline & (PC) SCR+FF+WFGD & 0 & 1 & 37 & 61 & 2 \\
\hline \multirow{8}{*}{$\mathrm{Pb} / \mathrm{Zn} / \mathrm{Cu}$ smelting $5,8,14$} & None & $3 / 3 / 0$ & $0 / 0 / 0$ & 34 & 56 & 10 \\
\hline & $\mathrm{DC}$ & $6 / 3 / 0$ & $0 / 0 / 0$ & 33 & 62 & 5 \\
\hline & $\mathrm{DC}+\mathrm{FGS}$ & $16 / 2 / 0$ & $10 / 4 / 0$ & 41 & 54 & 5 \\
\hline & $\mathrm{DC}+\mathrm{FGS}+\mathrm{ESD}+\mathrm{APS}$ & $22 / 19 / 2$ & $10 / 4 / 0$ & 57 & 38 & 5 \\
\hline & $\mathrm{DC}+\mathrm{FGS}+\mathrm{ESD}+\mathrm{APD}$ & $50 / 67 / 48$ & $50 / 40 / 40$ & 46 & 49 & 5 \\
\hline & DC+FGS+ESD+APD+DFGD & $0 / 0 / 33$ & $22 / 30 / 40$ & 94 & 6 & 0 \\
\hline & DC+FGS+ESD+APD+WFGD & $0 / 0 / 16$ & $5 / 10 / 20$ & 65 & 35 & 0 \\
\hline & DC+FGS+ESD+SMR+APD & $3 / 3 / 0$ & $3 / 12 / 0$ & 6 & 90 & 4 \\
\hline \multirow{3}{*}{ Cement production ${ }^{5,12}$} & $\begin{array}{l}\text { Shaft kiln/rotary kiln technology without } \\
\text { dust recycling }\end{array}$ & 9 & 2 & 80 & 15 & 5 \\
\hline & $\begin{array}{l}\text { Dry-process precalciner technology with } \\
\text { dust recycling }\end{array}$ & 61 & 8 & 24 & 75 & 1 \\
\hline & $\begin{array}{l}\text { Dry-process precalciner technology with } \\
\text { dust recycling and SNCR/SCR }\end{array}$ & 30 & 90 & 48 & 51 & 1 \\
\hline \multirow{6}{*}{ Iron and steel production ${ }^{15}$} & (Roasting plant) ESP & 50 & 50 & 14 & 85 & 1 \\
\hline & (Roasting plant) FF & 50 & 50 & 48 & 52 & 0 \\
\hline & (Sinter/Pellet plant) ESP & 47 & 28 & 18 & 82 & 0 \\
\hline & (Sinter/Pellet plant) FF & 40 & 35 & 18 & 82 & 0 \\
\hline & (Sinter/Pellet plant) ESP+WFGD & 13 & 27 & 41 & 59 & 0 \\
\hline & (Sinter/Pellet plant) COA+ESP+WFGD & 0 & 1 & 41 & 59 & 0 \\
\hline Coking ${ }^{15}$ & Wet & 50 & 100 & 56 & 44 & 1 \\
\hline Coal gasification ${ }^{15}$ & Wet & 50 & 100 & 56 & 44 & 1 \\
\hline
\end{tabular}

Notes: CFB-FGD - circulating fluidized bed flue gas desulfurization; DC - dust collector; FGS - flue gas scrubber; ESD - electrostatic demister; APS - acid plant with single contact and single absorption towers; APD 
- acid plant with double contact and double absorption towers; DFGD - dry flue gas desulfurization; SMR specific mercury removal devices; COA - catalytic oxidation and absorption for denitrogenation.

\section{Mercury Removal Efficiency by Typical APCD Combinations}

Table S6 shows the APCDs' removal efficiencies and the uncertainty bounds for coal-fired power plants, and details can be seen in Liu et al. (2018), Zhang et al (2012) and Zhang et al (2015). ${ }^{8,13,16}$ Table $\mathbf{S} 7$ shows the APCDs' removal efficiencies and the uncertainty bounds for industrial coal-fired boilers, heating supply, other coal combustion, $\mathrm{Pb} / \mathrm{Zn} / \mathrm{Cu}$ smelting, cement clinker production, iron and steel production (\%). 5, 8, 14, 15, 28

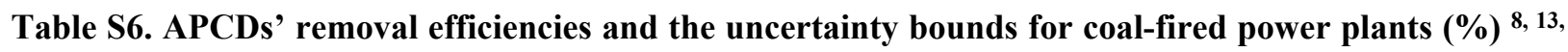

16

\begin{tabular}{cccccccc}
\hline $\begin{array}{c}\text { Boiler } \\
\text { type }\end{array}$ & APCDs type & Mean & Min & Max & SD & $\begin{array}{c}\text { Number } \\
\text { of tests }\end{array}$ & $\begin{array}{c}\text { Probabilistic } \\
\text { distribution }\end{array}$ \\
\hline PC & ESP+WFGD & 63.4 & 13.0 & 88.7 & 20.6 & 27 & Weibull \\
PC & SCR+ESP+WFGD & 70.4 & 37.0 & 90.0 & 12.8 & 16 & Weibull \\
PC & SCR+ESP+WFGD+WESP & 91.1 & 88.5 & 95.1 & 2.9 & 3 & Normal \\
PC & SCR+LTESP+WFGD+WESP & 91.1 & 88.5 & 95.1 & 2.9 & 3 & Normal \\
PC & FF+WFGD & 84.2 & 67.1 & 97.0 & 11.4 & 5 & Normal \\
PC & SCR+FF+WFGD & 87.8 & 76.3 & 99.3 & 11.5 & 2 & Normal \\
PC & SCR+ESP-FF+WFGD & 95.2 & 92.3 & 98.1 & 3.1 & 3 & Normal \\
PC & ESP+CFB-FGD+FF & 66.0 & 66.0 & 66.0 & - & 1 & Normal \\
CFB & ESP & 73.1 & 65.9 & 83.9 & 7.8 & 5 & Normal \\
CFB & FF & 75.8 & 59.0 & 92.5 & 12.1 & 3 & Normal \\
CFB & SNCR+ESP+WFGD & 98.1 & 98.1 & 98.1 & - & 1 & Normal \\
\hline
\end{tabular}


Table S7. APCDs' removal efficiencies and the uncertainty bounds for industrial coal-fired boilers, $\mathrm{Pb} / \mathrm{Zn} / \mathrm{Cu}$ smelting, cement clinker production, iron and steel production (\%)

\begin{tabular}{|c|c|c|c|c|c|c|c|}
\hline Emission source & APCD type & Mean & Min & Max & $\mathrm{SD}$ & $\begin{array}{l}\text { Test } \\
\text { No. }\end{array}$ & $\begin{array}{c}\text { Probabilistic } \\
\text { distribution }\end{array}$ \\
\hline \multirow{14}{*}{$\begin{array}{l}\text { Industrial coal- } \\
\text { fired boilers, } \\
\text { heating supply, } \\
\text { other coal } \\
\text { combustion } 5,8\end{array}$} & (SF) CYC & 1 & 0.2 & 0.9 & 0.5 & 22 & Weibull \\
\hline & (SF) WET & 23 & 7 & 59 & 18 & 8 & Normal \\
\hline & $(\mathrm{SF})$ IDRD & 38 & 17 & 58.5 & 20.8 & 3 & Normal \\
\hline & $(\mathrm{SF}) \mathrm{ESP}$ & 32.4 & 1 & 99.6 & 22.9 & 69 & Weibull \\
\hline & (SF) FF & 67.2 & 9 & 92 & 30 & 10 & Normal \\
\hline & (SF) FF+WFGD & 86 & 76.9 & 97 & 10.2 & 3 & Normal \\
\hline & (CFB) ESP+NID & 40 & 40 & 40 & 0 & 1 & Normal \\
\hline & (CFB) ESP+WFGD & 60.3 & 13 & 88 & 21.6 & 31 & Weibull \\
\hline & (CFB) FF+NID & 80 & 80 & 80 & 0 & 1 & Normal \\
\hline & (CFB) FF+WFGD & 86 & 76.9 & 97 & 10.2 & 3 & Normal \\
\hline & (CFB) SCR+FF+WFGD & 87.8 & 76.3 & 99.3 & 16.3 & 2 & Normal \\
\hline & $(\mathrm{PC}) \mathrm{ESP}$ & 32.4 & 1 & 99.6 & 22.9 & 69 & Weibull \\
\hline & (PC) ESP+WFGD & 60.3 & 13 & 88 & 21.6 & 31 & Weibull \\
\hline & (PC) SCR+FF+WFGD & 87.8 & 76.3 & 99.3 & 16.3 & 2 & Normal \\
\hline \multirow{7}{*}{$\begin{array}{l}\mathrm{Pb} / \mathrm{Zn} / \mathrm{Cu} \\
\text { smelting } 5,14,28,29\end{array}$} & $\mathrm{DC}$ & 10.4 & 1.3 & 25.2 & 8.5 & 9 & Normal \\
\hline & $\mathrm{DC}+\mathrm{FGS}$ & 41 & 27 & 55 & 19.8 & 2 & Normal \\
\hline & $\mathrm{DC}+\mathrm{FGS}+\mathrm{ESD}+\mathrm{APS}$ & 86.5 & 86.5 & 85.5 & 0 & 1 & Normal \\
\hline & $\mathrm{DC}+\mathrm{FGS}+\mathrm{ESD}+\mathrm{APD}$ & 97.4 & 87 & 100 & 5.1 & 6 & Normal \\
\hline & DC+FGS+ESD+APD+DFGD & 97.4 & 87 & 100 & 5.1 & 6 & Normal \\
\hline & DC+FGS+ESD+APD+WFGD & 98.5 & 98.5 & 98.5 & 0 & 1 & Normal \\
\hline & $\mathrm{DC}+\mathrm{FGS}+\mathrm{ESD}+\mathrm{SMR}+\mathrm{APD}$ & 99.2 & 99.1 & 99.3 & 0.1 & 2 & Normal \\
\hline \multirow{3}{*}{$\begin{array}{l}\text { Cement clinker } \\
\text { production }\end{array}$} & $\begin{array}{l}\text { Shaft kiln/rotary kiln technology } \\
\text { without dust recycling }\end{array}$ & 61.8 & 25 & 98 & 27.7 & 12 & Normal \\
\hline & $\begin{array}{l}\text { Dry-process precalciner } \\
\text { technology with dust recycling }\end{array}$ & 6.2 & 0.7 & 21.1 & 7.5 & 6 & Normal \\
\hline & $\begin{array}{l}\text { Dry-process precalciner } \\
\text { technology with dust recycling } \\
\text { and SNCR }\end{array}$ & 15 & 15 & 15 & 0 & 1 & Normal \\
\hline \multirow{6}{*}{$\begin{array}{l}\text { Iron and steel } \\
\text { production }^{15}\end{array}$} & (Roasting plant) ESP & 29 & 29 & 29 & 0 & 1 & Normal \\
\hline & (Roasting plant) FF & 67 & 67 & 67 & 0 & 1 & Normal \\
\hline & (Sinter/Pellet plant) ESP & 29 & 29 & 29 & 0 & 1 & Normal \\
\hline & (Sinter/Pellet plant) FF & 67 & 67 & 67 & 0 & 1 & Normal \\
\hline & (Sinter/Pellet plant) ESP+WFGD & 57 & 57 & 57 & 0 & 1 & Normal \\
\hline & $\begin{array}{l}\text { (Sinter/Pellet plant) } \\
\text { COA+ESP+WFGD }\end{array}$ & 72 & 72 & 72 & 0 & 1 & Normal \\
\hline Coking ${ }^{15}$ & Wet & 85 & 85 & 85 & 0 & 1 & Normal \\
\hline $\begin{array}{l}\text { Coal } \\
\text { gasification }{ }^{15}\end{array}$ & Wet & 85 & 85 & 85 & 0 & 1 & Normal \\
\hline
\end{tabular}




\section{Methodology for Uncertainty Analysis}

Monte Carlo simulations were used to analyze the uncertainty of 29 categories' mercury emissions by taking into account the probability distribution of key parameters. Normal distribution with a coefficient of variation $(\mathrm{CV}$, the standard deviation divided by the mean) of $5 \%$ was assumed for activity levels. Mercury contents of fuel/raw material fitted log-normal distribution curve, shown in Table S3-S4. The distributions characteristics of APCDs' removal efficiencies were shown in Table S6-S7. Details of mercury contents and APCDs' removal efficiencies can be seen in Zhang et al. (2015), Wu et al. (2016), Wu et al. (2017) and Liu et al. (2018).5, 8, 15, 16 We then ran the simulations for 10000 times and got the results in the form of a statistical distribution. The P10 and P90 values of the distribution curve were set as the lower and upper limit of the simulation results. The (P50-P10)/P50 and (P90-P50)/P50 values were the lower and upper limit of the uncertainty range with a confidence level of $80 \%$.

In 2017, the national annual anthropogenic mercury emissions were estimated to be 444 tons, including 244 tons of GEM, 183 tons of GOM and 17 tons of PBM. Monte Carlo simulations were used to produce the probabilistic emissions by taking into account key parameters in eq.1, including the probability distribution of mercury concentration in fuel/raw materials and mercury removal efficiencies of APCDs. $5,8,13,15,16$ The overall uncertainty was estimated to range from $-20 \%$ to $23 \%$. Thereinto, 129 tons $(-65 \%, 75 \%)$ of mercury were emitted from cement clinker production, 48 tons $(-19 \%, 21 \%)$ from coal-fired power plants, 39 tons $(-45 \%, 49 \%)$ from industrial coal boilers, 38 tons $(-55 \%, 62 \%)$ from $\mathrm{Zn}$ smelting, 32 tons $(-58 \%, 70 \%)$ from iron and steel production, 27 tons $(-65 \%, 72 \%)$ from $\mathrm{Pb}$ smelting, 27 tons $(-20 \%, 22 \%)$ from heating supply, 27 tons $(-20 \%$, $23 \%)$ from residential coal combustion, 13 tons $(-50 \%, 50 \%)$ from municipal solid waste incineration, 13 tons ($56 \%, 67 \%)$ from $\mathrm{Al}$ smelting, 14 tons $(-20 \%, 23 \%)$ from other coal combustion. The details for uncertainty ranges of all the sectors are illustrated in Figure S5. 


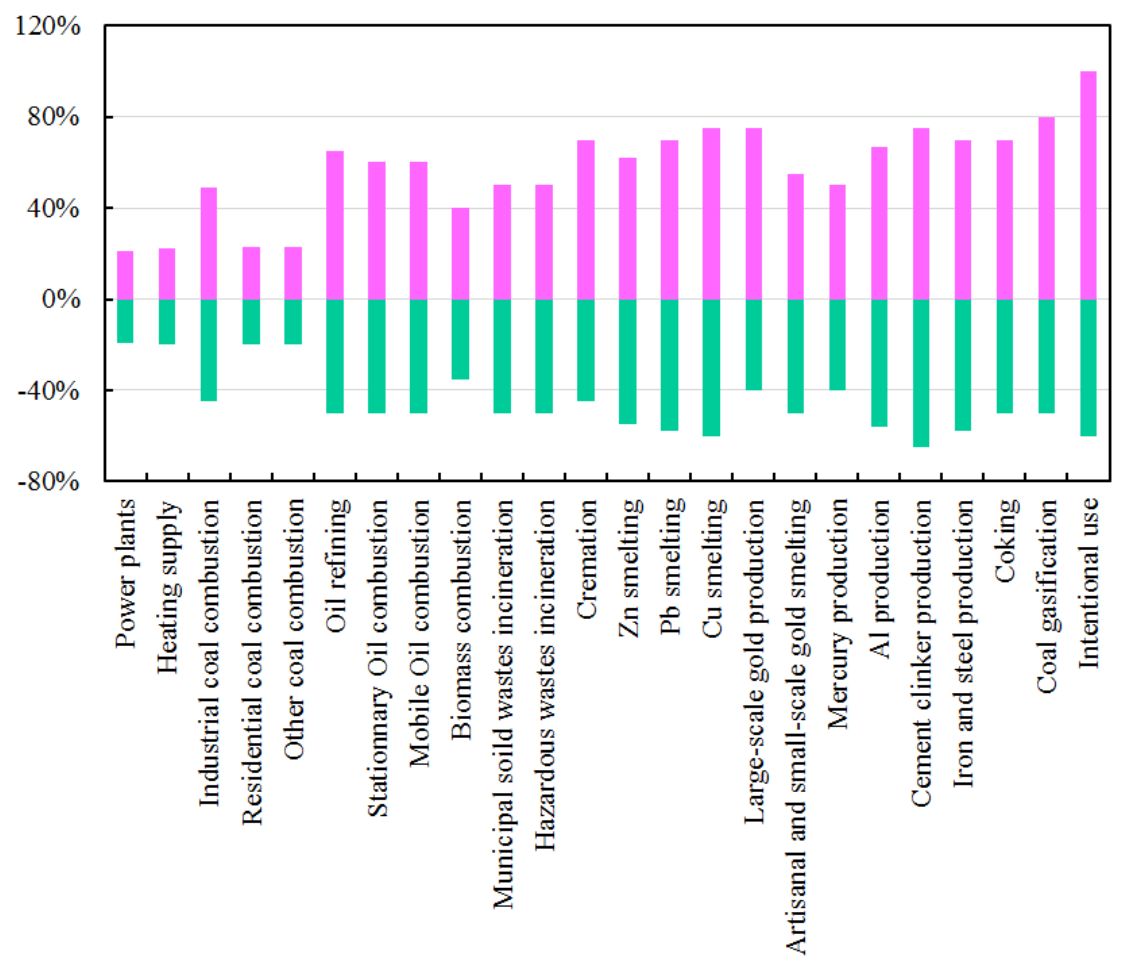

Figure S5. Uncertainty ranges of mercury emissions from different sources in China, 2017

\section{Gridded Natural Mercury Emissions in China}

With the input of updated mercury concentration in surface soil $(0-20 \mathrm{~cm})$ of China in Wang et al. (2016), the simulated mercury emission from surface soil by the nested-grid GEOS-Chem model for China domain is shown in Figure S6. ${ }^{30}$

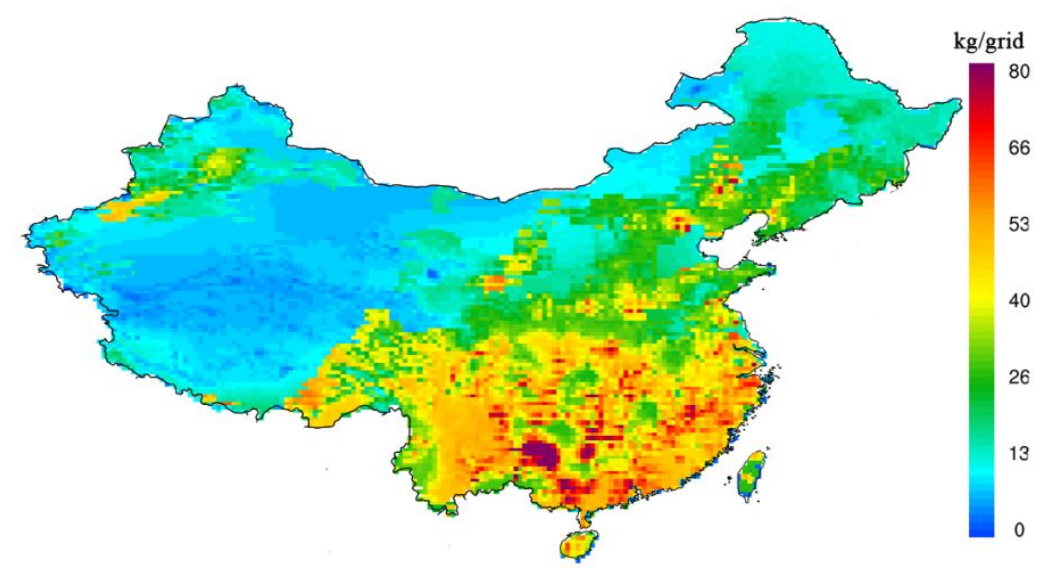

Figure S6. Gridded natural mercury emissions in China 


\section{Gridded Emission Inventory}

GEM, GOM and PBM emissions from anthropogenic sources are gridded to provide input for GEOS-ChemCH model, which covers the years between 2013 and 2017. Figure S7 shows the gridded anthropogenic mercury emissions in China for the year of 2013 and 2017. Anthropogenic mercury emissions are concentrated in central and eastern China. In BTH, YRD, SC and HHJ, the mean mercury emission intensities are over $100 \mathrm{~g} / \mathrm{km}^{2}$. The results of the mercury emissions in 2017 may serve as the baseline for the convention implementation.

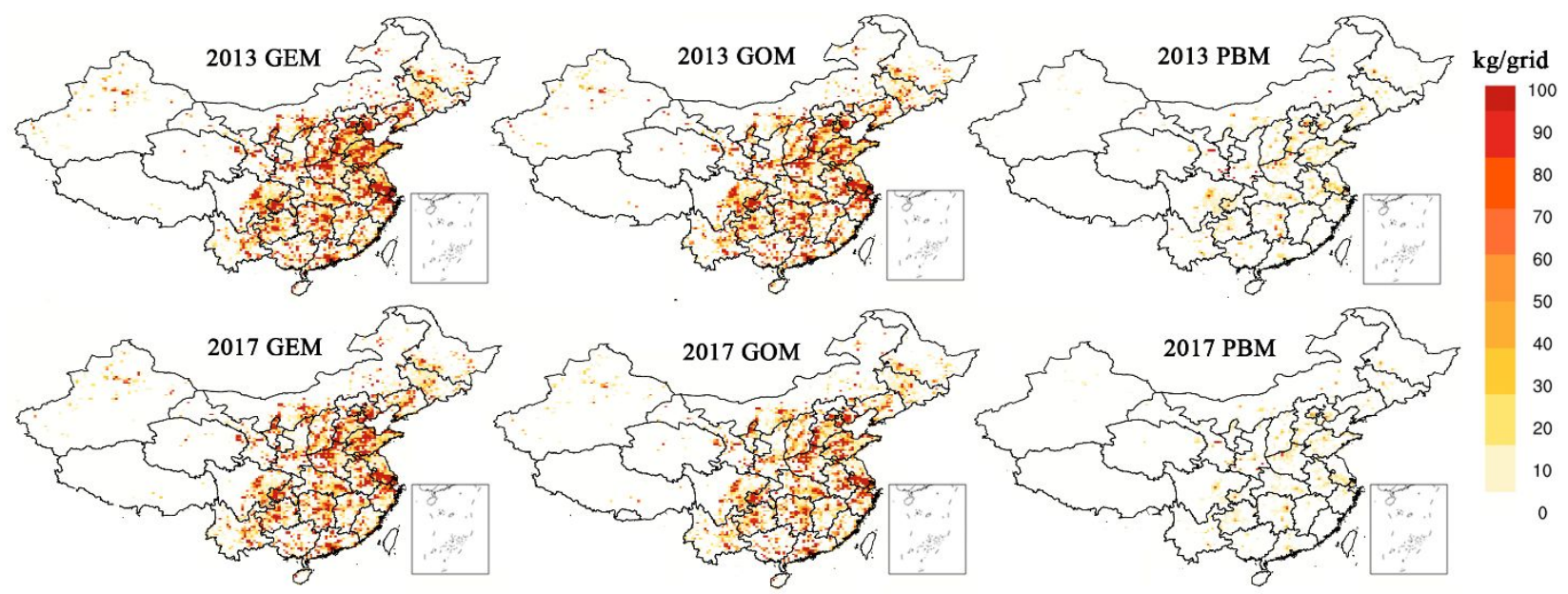

Figure S7. Gridded anthropogenic mercury emissions in China during 2013-2017

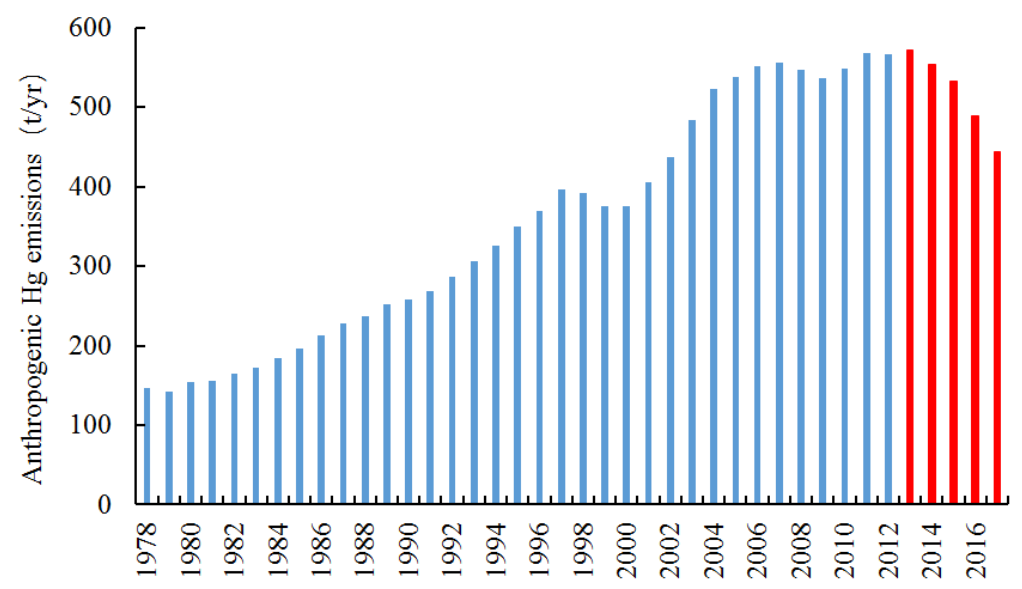

Figure S8. Anthropogenic mercury emissions during 1978-2017 in China

\section{Observed TGM Concentrations to Evaluate the Performance of GEOS-Chem-CH Model}


Table S8 illustrates the statistical summary of TGM concentrations and associated site information of 12 rural or remote ground stations in China, which is used to evaluate the model performance. Figure S9 shows the comparison of simulated and observed annual or seasonal mean TGM concentrations for 12 rural or remote ground stations in China. Figure S10 shows the comparison of simulated and observed wet deposition flux in 17 rural or remote ground stations and dry deposition flux in 6 rural or remote ground stations in Table S9. In the GEOS-Chem, mercury emissions from all sources are instantaneously diluted in grid cells, so the simulation represent the mean mercury concentrations and depositions of grid cells. However, urban sites' observations are usually under grate fluctuation due to strong anthropogenic activities. Therefore, comparisons with urban sites' observations are not included, mainly because these observations may not well represent the mean mercury concentrations of grid cells where they locate. The rural or remote ground sites for model evaluation in this study are far from anthropogenic activities and less influenced by local sources, which can represent the background mercury levels in Chinese mainland. In addition, the model performance in urban areas was also crucial to the evaluation, which hasn't been included in this study. The mean reason was that few observations at Chinese urban sites during 2013-2017 was found. In the near future, we will improve the performance of nested GEOSChem-CH model under high urban PM2.5 concentrations through changing the gas-particle distribution of GOM.

Table S8. TGM concentrations at rural or remote ground stations in China

\begin{tabular}{|c|c|c|c|c|c|c|}
\hline \multirow{2}{*}{ Site } & \multicolumn{2}{|c|}{ Location } & \multirow{2}{*}{$\begin{array}{l}\text { Altitude } \\
\text { (m a.s.l) }\end{array}$} & \multirow{2}{*}{ Monitoring period } & \multirow{2}{*}{$\begin{array}{c}\text { TGM } \\
\left(\mathrm{ng} / \mathrm{m}^{3}\right) \\
\end{array}$} & \multirow{2}{*}{ Reference } \\
\hline & Lon $(\mathrm{E})$ & Lat $(\mathrm{N})$ & & & & \\
\hline Mt. Damei & $121.565^{\circ}$ & $29.632^{\circ}$ & 550 & $4 / 2011-4 / 2013$ & $3.46 \pm 1.44$ & Fu et al., $(2015)^{31}$ \\
\hline Mt. Waliguan & $100.898^{\circ}$ & $36.287^{\circ}$ & 3816 & $9 / 2007-9 / 2008$ & $2.01 \pm 0.98$ & Fu et al., (2012) 32 \\
\hline Shangri-La & $99.733^{\circ}$ & $28.017^{\circ}$ & 3580 & 11/2009-11/2010 & $2.59 \pm 2.73$ & Zhang et al., (2015) 33 \\
\hline Mt. Leigong & $108.20^{\circ}$ & $26.39^{\circ}$ & 2178 & $5 / 2008-5 / 2009$ & $2.80 \pm 1.51$ & Fu et al., $(2010)^{34}$ \\
\hline Chengshantou & $122.68^{\circ}$ & $37.38^{\circ}$ & 30 & 1/2009-10/2009 & $2.31 \pm 0.74$ & Ci et al., (2011) ${ }^{35}$ \\
\hline Nam Co Lake & $90.989^{\circ}$ & $30.774^{\circ}$ & 4730 & $11 / 2014-2 / 2015$ & $0.95 \pm 0.37$ & Foy et al , (2016) 36 \\
\hline Qingdao & $120.5^{\circ}$ & $36.16^{\circ}$ & 40 & $1 / 2013$ & $3.04 \pm 0.9$ & Zhang et al., (2014) 37 \\
\hline Mt. Wuzhi & $109.492^{\circ}$ & $18.836^{\circ}$ & 958 & $5 / 2011-5 / 2012$ & $1.58 \pm 0.71$ & Liu et al, (2016) ${ }^{38}$ \\
\hline Mt. Wuzhi & $109.493^{\circ}$ & $18.837^{\circ}$ & 958 & 9/2011-11/2011 & $1.86 \pm 0.55$ & Liu et al, (2016) ${ }^{38}$ \\
\hline Mt. Wuzhi & $109.494^{\circ}$ & $18.838^{\circ}$ & 958 & $12 / 2011-2 / 2012$ & $1.8 \pm 0.62$ & Liu et al, (2016) ${ }^{38}$ \\
\hline Mt. Wuzhi & $109.495^{\circ}$ & $18.839^{\circ}$ & 958 & $3 / 2012-5 / 2012$ & $1.16 \pm 0.45$ & Liu et al, (2016) ${ }^{38}$ \\
\hline Mt. Wuzhi & $109.496^{\circ}$ & $18.840^{\circ}$ & 958 & $7 / 2011-9 / 2011$ & $1.43 \pm 0.46$ & Liu et al, (2016) ${ }^{38}$ \\
\hline Mt. Changbai & $128.112^{\circ}$ & $42.402^{\circ}$ & 740 & $1 / 2013-12 / 2015$ & $1.64 \pm 0.46$ & Fu et al., (2016) ${ }^{39}$ \\
\hline Mt. Ailao & $101.017^{\circ}$ & $24.533^{\circ}$ & 2450 & $5 / 2011-5 / 2012$ & $2.09 \pm 0.63$ & Zhang et al., (2015) 40 \\
\hline
\end{tabular}




\begin{tabular}{lccclll} 
Mt. Ailao & $101.017^{\circ}$ & $24.533^{\circ}$ & 2450 & $3 / 2012-5 / 2012$ & $2.18 \pm 0.67$ & Zhang et al., (2015) ${ }^{40}$ \\
Mt. Ailao & $101.017^{\circ}$ & $24.533^{\circ}$ & 2450 & $6 / 2011-8 / 2011$ & $2.20 \pm 0.60$ & Zhang et al., (2015) \\
Mt. Ailao & $101.017^{\circ}$ & $24.533^{\circ}$ & 2450 & $9 / 2011-11 / 2011$ & $1.92 \pm 0.64$ & Zhang et al., (2015) \\
Mt. Ailao & $101.017^{\circ}$ & $24.533^{\circ}$ & 2450 & $12 / 2011-2 / 2012$ & $2.04 \pm 0.58$ & Zhang et al., (2015) ${ }^{40}$ \\
Miyun & $116.775^{\circ}$ & $40.481^{\circ}$ & 220 & $7 / 2012-6 / 2013$ & $2.67 \pm 1.48$ & This study \\
Chongming & $121.908^{\circ}$ & $31.522^{\circ}$ & 11 & $11 / 2014-10 / 2015$ & $2.13 \pm 0.66$ & This study \\
\hline
\end{tabular}

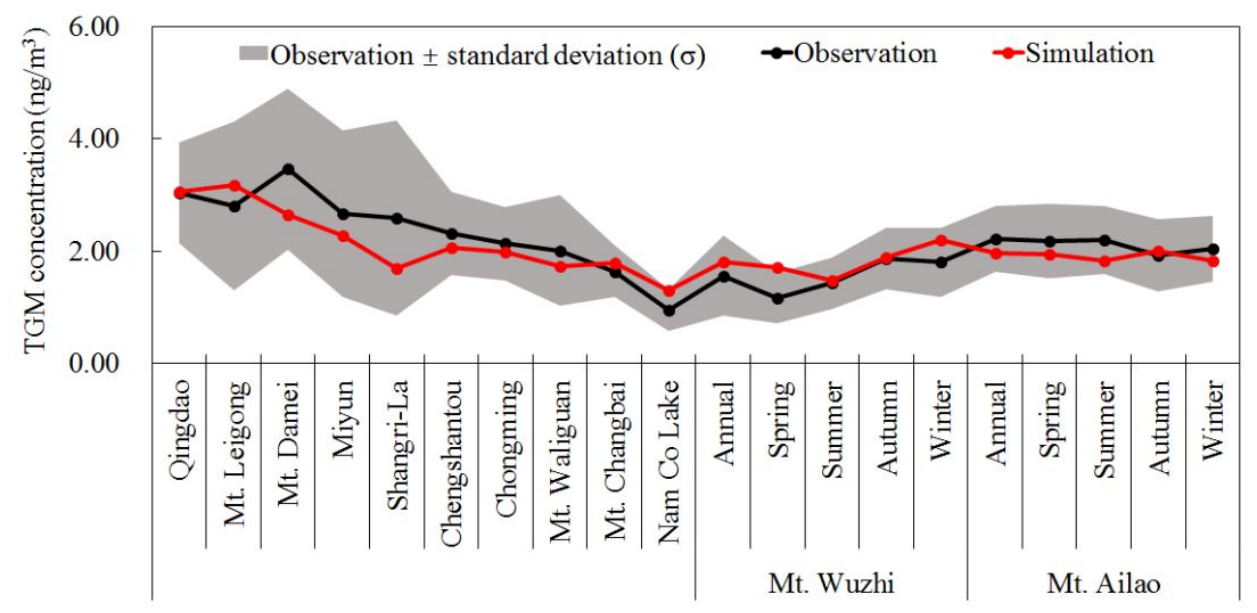

Figure S9. Comparison of simulated and observed annual or seasonal mean TGM concentrations

Table S9. Observed atmospheric mercury deposition flux in remote stations for model evaluation

\begin{tabular}{|c|c|c|c|c|c|c|c|}
\hline \multirow{2}{*}{ Sites } & \multicolumn{2}{|c|}{ Location } & \multirow{2}{*}{$\begin{array}{l}\text { Altitude } \\
\text { (m a.s.l) }\end{array}$} & \multirow{2}{*}{$\begin{array}{l}\text { Monitoring } \\
\text { period }\end{array}$} & \multicolumn{2}{|c|}{$\begin{array}{l}\text { Deposition flux } \\
\left(\mu \mathrm{g} \mathrm{m}^{-2} \mathrm{yr}^{-1}\right)\end{array}$} & \multirow{2}{*}{ Reference } \\
\hline & Lon (E) & Lat $(\mathrm{N})$ & & & Wet & Dry & \\
\hline Mt. Ailao & $101.02^{\circ}$ & $24.532^{\circ}$ & 2500 & $6 / 2011-5 / 2014$ & 7.2 & 62.8 & Fu et al. $(2016)^{41}$ \\
\hline Mt. Leigong & $108.2^{\circ}$ & $26.392^{\circ}$ & 2178 & $5 / 2008-5 / 2009$ & 6.1 & 43.9 & Fu et al. $(2011)^{34}$ \\
\hline Mt. Damei & $121.57^{\circ}$ & $29.632^{\circ}$ & 550 & $8 / 2012-7 / 2013$ & 7 & 23.1 & Lang $(2014)^{42}$ \\
\hline Nam Co Lake & $90.99^{\circ}$ & $30.772^{\circ}$ & 4730 & $7 / 2009-7 / 2011$ & 1.75 & & Huang et al. (2012) 43 \\
\hline Mt. Gongga & $102.12^{\circ}$ & $29.652^{\circ}$ & 1640 & $1 \sim 12 / 2006$ & 9.1 & & Fu et al. (2008) ${ }^{44}$ \\
\hline Mt. Gongga & $101.93^{\circ}$ & $29.582^{\circ}$ & 3000 & $5 / 2005-4 / 2005$ & 26.1 & 66.5 & Fu et al. (2010) ${ }^{45}$ \\
\hline Mt. Changbai & $128.47^{\circ}$ & $42.42^{\circ}$ & 750 & $8 / 2005-7 / 2006$ & 8.4 & & Wan et al. (2009) 46 \\
\hline Puding & $105.8^{\circ}$ & $26.372^{\circ}$ & 1145 & $8 / 2005-7 / 2006$ & 24.8 & & Guo et al. (2008) 47 \\
\hline Hongjiadu & $105.85^{\circ}$ & $26.882^{\circ}$ & 1130 & $8 / 2005-7 / 2006$ & 34.7 & & Guo et al. $(2008)^{47}$ \\
\hline Yinzidu & $106.12^{\circ}$ & $26.572^{\circ}$ & 1088 & $8 / 2005-7 / 2006$ & 38.1 & & Guo et al. (2008) 47 \\
\hline Dongfeng & $106.13^{\circ}$ & $26.852^{\circ}$ & 970 & $8 / 2005-7 / 2006$ & 36.3 & & Guo et al. (2008) ${ }^{47}$ \\
\hline Wujiangdu & $106.77^{\circ}$ & $27.322^{\circ}$ & & $8 / 2005-7 / 2006$ & 39.6 & & Guo et al. (2008) ${ }^{47}$ \\
\hline Mt. Leigong & $108.2^{\circ}$ & $26.392^{\circ}$ & 2178 & $3 / 2005-2 / 2006$ & 19 & 100.5 & Guo et al. (2008) ${ }^{47}$ \\
\hline Mt. Changbai & $128.11^{\circ}$ & $42.40^{\circ}$ & 736 & $8 / 2011-8 / 2014$ & 5.6 & 22.8 & Fu et al. (2016) ${ }^{41}$ \\
\hline Mt. Waliguan & $100.898^{\circ}$ & $36.287^{\circ}$ & 3816 & $5 / 2012-8 / 2014$ & 2.0 & & Fu et al. (2016) ${ }^{41}$ \\
\hline Bayinbuluk & $83.72^{\circ}$ & $42.89^{\circ}$ & 2500 & $10 / 2013-10 / 2014$ & 2.0 & & Fu et al. (2016) ${ }^{41}$ \\
\hline
\end{tabular}




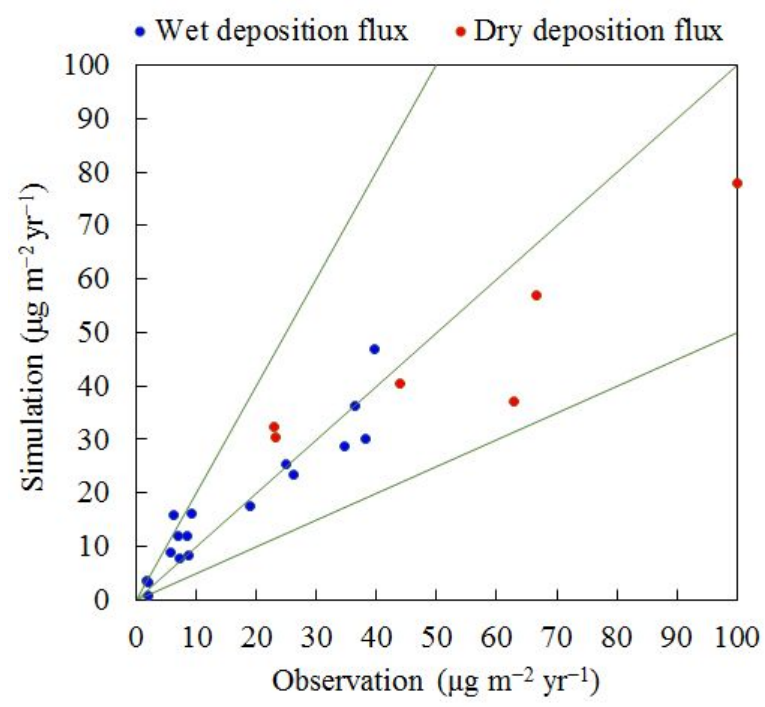

Figure S10. Comparison of simulated and observed annual wet/dry deposition flux in remote stations

\section{Changes of Anthropogenic Mercury Emissions during 2013-2017}

Table S10. Major sources' emission reductions in different domains during 2013-2017 (tons)

\begin{tabular}{|c|c|c|c|c|c|c|c|c|c|}
\hline $\begin{array}{c}\text { Major } \\
\text { Sources }\end{array}$ & Measures & China & North & Northeast & East & Central & South & Southwest & Northwes \\
\hline \multirow{4}{*}{$\begin{array}{l}\text { Coal-fired } \\
\text { power } \\
\text { plants }\end{array}$} & Energy conservation & 7.3 & -1.1 & 1.0 & 0.5 & 1.4 & 0.71 & 3.80 & 1.04 \\
\hline & $\begin{array}{l}\text { Ultra-low emission } \\
\text { renovation }\end{array}$ & 44.5 & 13.2 & 4.8 & 14.6 & 2.2 & 2.9 & 2.6 & 4.2 \\
\hline & Newly-built SCR devices & 4.9 & 1.5 & 0.5 & 1.6 & 0.2 & 0.3 & 0.3 & 0.5 \\
\hline & Subtotal & 56.8 & 13.6 & 6.4 & 16.7 & 3.8 & 3.9 & 6.7 & 5.7 \\
\hline \multirow{5}{*}{$\begin{array}{l}\text { Industrial } \\
\text { coal } \\
\text { combustion }\end{array}$} & Energy conservation & -4.3 & -0.3 & -0.8 & 2.2 & 0.3 & -0.6 & -0.2 & -4.8 \\
\hline & $\begin{array}{l}\text { Upgrading dedusting } \\
\text { devices }\end{array}$ & 11.6 & 2.3 & 1.4 & 2.3 & 1.6 & 0.7 & 1.6 & 1.6 \\
\hline & Newly-built FGD devices & 19.9 & 3.9 & 2.4 & 4.0 & 2.7 & 1.2 & 2.8 & 2.8 \\
\hline & Newly-built SCR devices & 1.7 & 0.3 & 0.2 & 0.3 & 0.2 & 0.1 & 0.2 & 0.2 \\
\hline & Subtotal & 28.8 & 6.2 & 3.2 & 8.9 & 4.8 & 1.5 & 4.5 & -0.2 \\
\hline \multirow{3}{*}{$\begin{array}{l}\text { Cement } \\
\text { clinker } \\
\text { production }\end{array}$} & Cutting back production & 2.4 & 2.7 & 1.5 & 0.5 & 2.3 & -1.3 & -2.1 & -1.3 \\
\hline & $\begin{array}{l}\text { Newly-built SCR/SNCR } \\
\text { devices }\end{array}$ & 11.4 & 1.8 & 0.3 & 2.6 & 3.7 & -0.4 & -0.1 & 3.7 \\
\hline & Subtotal & 13.8 & 4.5 & 1.8 & 3.1 & 6.0 & -1.8 & -2.2 & 2.4 \\
\hline \multirow{3}{*}{$\begin{array}{l}\text { Non- } \\
\text { ferrous } \\
\text { metal } \\
\text { smelting }\end{array}$} & Cutting back production & -23.5 & -2.9 & -0.1 & -0.7 & -6.7 & -1.93 & -2.04 & -9.08 \\
\hline & Newly-built FGD devices & 41.9 & 4.5 & 0.7 & 2.2 & 23.3 & 2.8 & 6.4 & 2.1 \\
\hline & Subtotal & 18.5 & 1.6 & 0.6 & 1.5 & 16.6 & 0.8 & 4.4 & -7.0 \\
\hline
\end{tabular}




\begin{tabular}{llcccccccc}
\hline Iron and & Cutting back production & 1.4 & -0.2 & -0.3 & 0.7 & -0.1 & -0.16 & 1.04 & 0.46 \\
steel & Newly-built FGD devices & 6.9 & 2.5 & 0.7 & 1.7 & 0.6 & 0.3 & 0.4 & 0.7 \\
production & Subtotal & 8.3 & 2.3 & 0.4 & 2.4 & 0.5 & 0.2 & 1.4 & 1.1 \\
\multirow{2}{*}{ Others } & Coal shift to gas & 0.8 & 1.3 & 0.4 & -0.3 & -0.2 & 0.4 & -0.2 & -0.6 \\
& Subtotal & 0.8 & 1.3 & 0.4 & -0.3 & -0.2 & 0.4 & -0.2 & -0.6 \\
\hline
\end{tabular}

\section{Measure-Specific Impacts on Mercury Mass-balance}

(a)

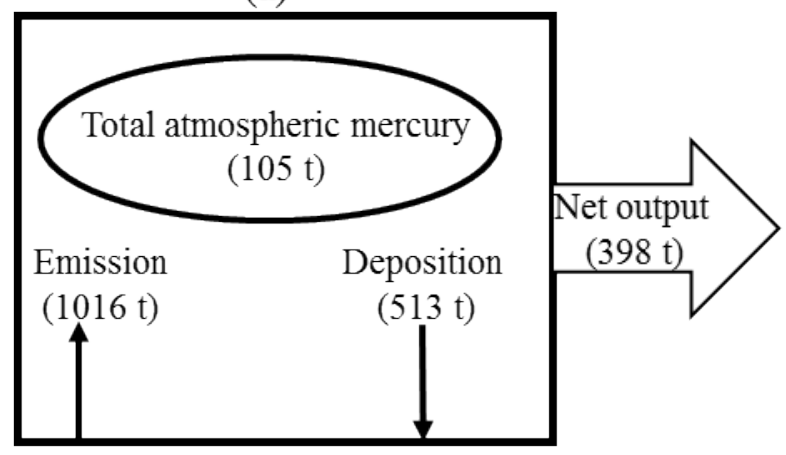

(b)

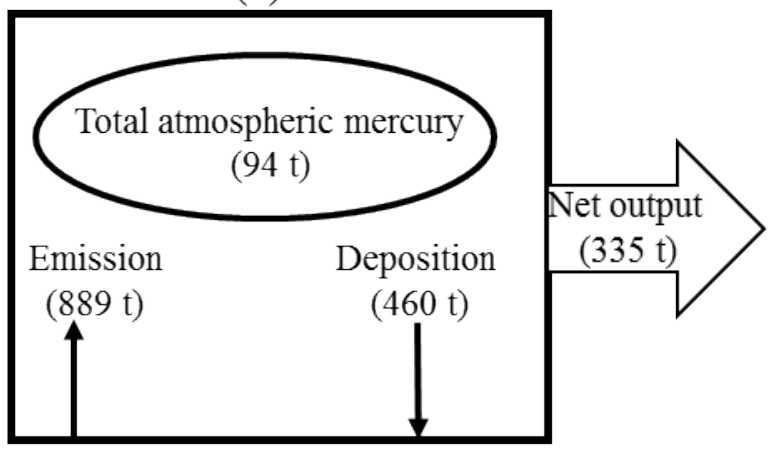

Figure S11. Mercury mass-balance in BASE scenario with the input of anthropogenic mercury emissions and meteorology conditions in 2013 (a); Mercury mass-balance in BASE scenario in CTRL scenario with the input of anthropogenic mercury emissions in 2017 and meteorology conditions in 2013 (b)

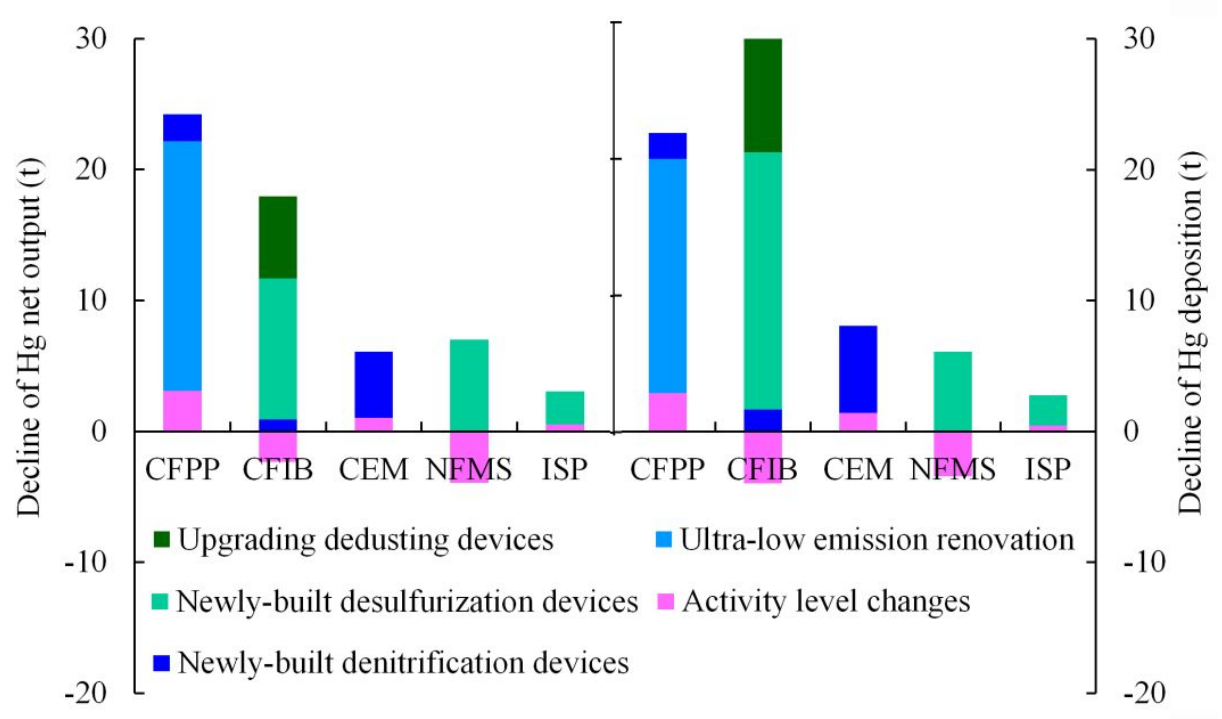

Figure S12. Measure-specific benefits on the declines of mercury net output and deposition 


\section{Measure-Specific Improvements of Atmospheric Mercury Pollution}

Table S11. Measure-specific benefits for the mitigated mercuey emissions $\left(\Delta \mathrm{EMIS}_{c, j}\right)$, TGM concentration $\left(\Delta \operatorname{TGM}_{c, j}\right), \operatorname{FLX}\left(\Delta \operatorname{FLX}_{c, j}\right), \operatorname{DFLX}\left(\Delta \operatorname{DFLX}_{c, j}\right)$ and WFLX $\left(\Delta \operatorname{WFLX}_{c, j}\right)$ during 2013-2017

\begin{tabular}{|c|c|c|c|c|c|c|}
\hline $\begin{array}{c}\text { Major } \\
\text { Sources }(j)\end{array}$ & Measures $(c)$ & $\begin{array}{c}\Delta \mathrm{EMIS}_{c, j} \\
(\mathrm{t})\end{array}$ & $\begin{array}{l}\Delta \mathrm{TGM}_{c, j} \\
\left(\mathrm{ng} \mathrm{m}^{-3}\right)\end{array}$ & $\begin{array}{c}\Delta \mathrm{FLX}_{c, j} \\
\left(\mu \mathrm{g} \mathrm{m}^{-2} \mathrm{yr}^{-1}\right)\end{array}$ & $\begin{array}{c}\Delta \mathrm{DFLX}_{c, j} \\
\left(\mu \mathrm{g} \mathrm{m}^{-2} \mathrm{yr}^{-1}\right)\end{array}$ & $\begin{array}{c}\Delta \mathrm{WFLX}_{c, j} \\
\left(\mu \mathrm{g} \mathrm{m}^{-2} \mathrm{yr}^{-1}\right)\end{array}$ \\
\hline \multirow{4}{*}{$\begin{array}{l}\text { Coal-fired } \\
\text { power } \\
\text { plants }\end{array}$} & Energy conservation & 7.3 & 0.02 & 0.74 & 0.48 & 0.26 \\
\hline & Ultra-low emission renovation & 44.5 & 0.11 & 4.52 & 2.95 & 1.57 \\
\hline & Newly-built SCR devices & 4.9 & 0.01 & 0.50 & 0.32 & 0.17 \\
\hline & Subtotal & 56.8 & 0.14 & 5.76 & 3.76 & 2.00 \\
\hline \multirow{5}{*}{$\begin{array}{l}\text { Industrial } \\
\text { coal } \\
\text { combustion }\end{array}$} & Energy conservation & -4.3 & -0.01 & -1.07 & -0.66 & -0.42 \\
\hline & Upgrading dedusting devices & 11.6 & 0.01 & 0.42 & 0.26 & 0.16 \\
\hline & Newly-built FGD devices & 19.9 & 0.06 & 4.96 & 3.04 & 1.93 \\
\hline & Newly-built SCR devices & 1.7 & 0.03 & 2.89 & 1.77 & 1.12 \\
\hline & Subtotal & 28.8 & 0.09 & 7.21 & 4.41 & 2.80 \\
\hline \multirow{3}{*}{$\begin{array}{l}\text { Cement } \\
\text { clinker } \\
\text { production }\end{array}$} & Cutting back production & 2.4 & 0.01 & 0.35 & 0.22 & 0.14 \\
\hline & Newly-built SCR/SNCR devices & 11.4 & 0.03 & 1.69 & 1.04 & 0.65 \\
\hline & Subtotal & 13.8 & 0.04 & 2.04 & 1.26 & 0.78 \\
\hline \multirow{3}{*}{$\begin{array}{l}\text { Non- } \\
\text { ferrous } \\
\text { metal } \\
\text { smelting }\end{array}$} & Cutting back production & -23.5 & -0.02 & -0.86 & -0.51 & -0.35 \\
\hline & Newly-built FGD devices & 41.9 & 0.04 & 1.54 & 0.92 & 0.62 \\
\hline & Subtotal & 18.5 & 0.02 & 0.68 & 0.40 & 0.27 \\
\hline \multirow{3}{*}{$\begin{array}{l}\text { Iron and } \\
\text { steel } \\
\text { production }\end{array}$} & Cutting back production & 1.4 & 0.00 & 0.12 & 0.08 & 0.04 \\
\hline & Newly-built FGD devices & 6.9 & 0.01 & 0.59 & 0.37 & 0.22 \\
\hline & Subtotal & 8.3 & 0.01 & 0.70 & 0.44 & 0.26 \\
\hline
\end{tabular}



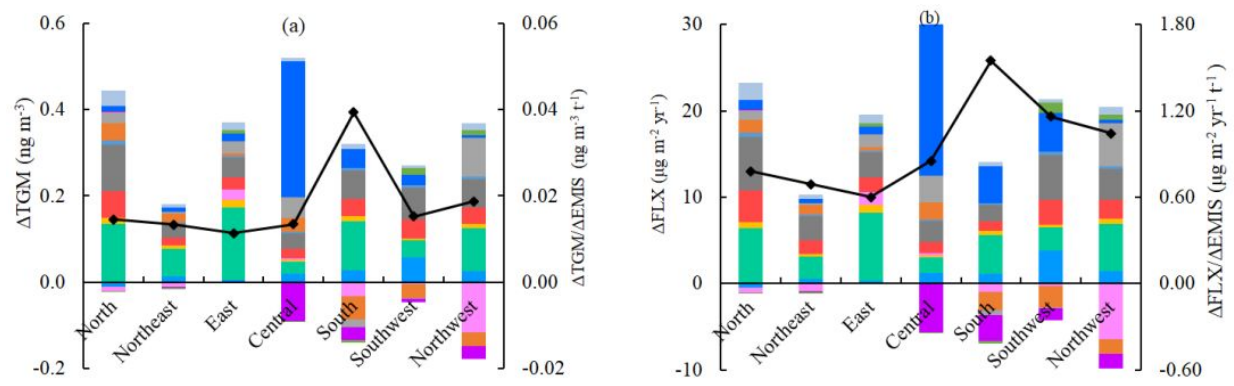

$=$ Newly-built WFGD devices in ISP

-Cutting back production in ISP

- Newly-built FGD devices in NFMS

- Cutting back production in NFMS

- Newly-built SCR/SNCR devices in CEM

- Cutting back production in CEM

-Newly-built SCR devices in CFIB

-Newly-built WFGD devices in CFIB

$=$ Upgrading dedusting devices in CFIB
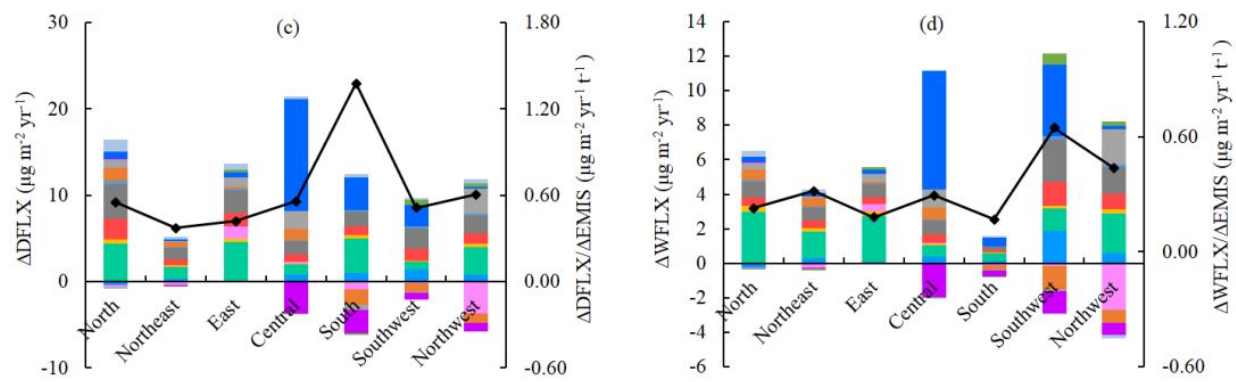

Energy conservation in CFIB

$=$ Newly-built SCR devices in CFPP

-Ultra-low emission renevation in CFPP

Energy conservation in CFPP

$\rightarrow \Delta$ TGM/ $\triangle \mathrm{EMIS}, \Delta \mathrm{FLX} / \triangle \mathrm{EMIS}$, $\triangle \mathrm{DFLX} / \triangle \mathrm{EMIS}, \Delta \mathrm{WFLX} / \triangle \mathrm{EMIS}$

Figure S13. Measure-specific improvements of atmospheric mercury pollution in different regions

\section{Measure-Specific Benefits of Unit Emissions Reductions}

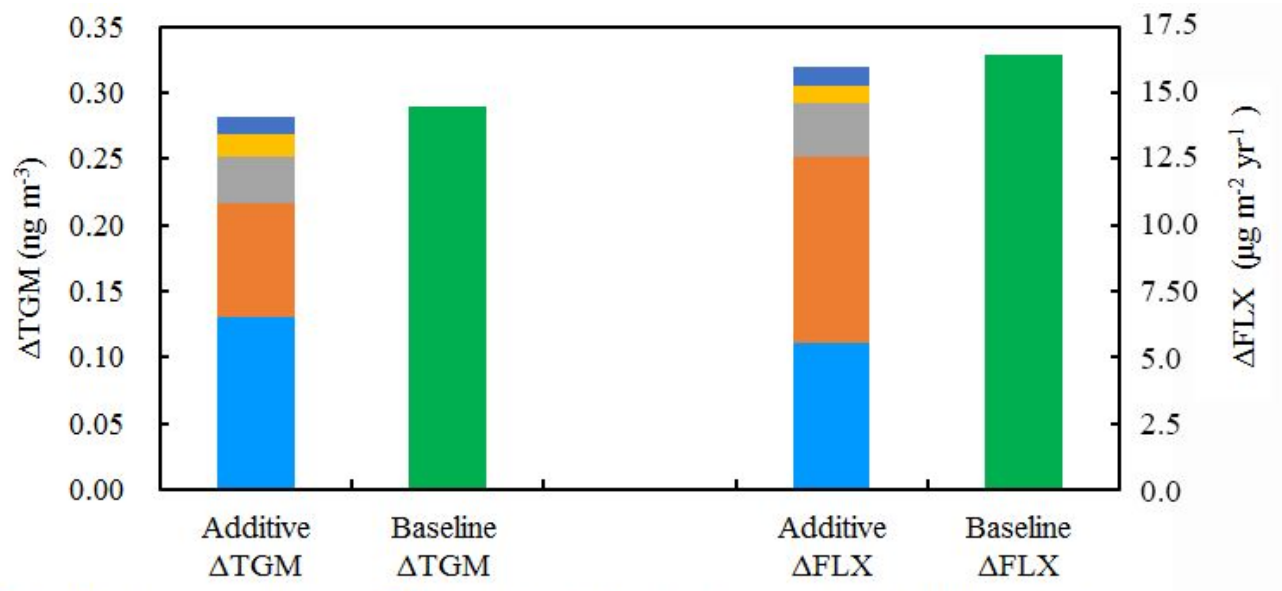

a Coal-fired power plants $\quad$ Industrial coal combustion $\quad$ Cement clinker production

Non-ferrous metal smelting $\mathbf{m}$ Iron and steel production

Figure S14. Comparison of the additive $\Delta \mathrm{TGM}$ and $\triangle \mathrm{FLX}$ to the baseline levels 


\section{Policy Implications}

Table S12 Atmospheric mercury pollution in 2017 and mitigation for 2013-2017

\begin{tabular}{ccccccc}
\hline \multirow{2}{*}{ Sectors } & \multicolumn{3}{c}{ Status in 2017 } & & \multicolumn{2}{c}{ Mitigation for 2013-2017 } \\
\cline { 2 - 4 } & $\begin{array}{c}\text { EMIS } \\
(\mathrm{t})\end{array}$ & $\begin{array}{c}\text { Contributions to TGM } \\
\left(\mathrm{ng} \mathrm{m}^{-3}\right)\end{array}$ & $\begin{array}{c}\text { Contributions to FLX } \\
\left(\mu \mathrm{g} \mathrm{m}^{-2} \mathrm{yr}^{-1}\right)\end{array}$ & & $\begin{array}{c}\Delta \text { TGM/ } / \Delta \text { EMIS } \\
\left(\mathrm{pg} \mathrm{m}^{-3} \mathrm{t}^{-1}\right)\end{array}$ & $\begin{array}{c}\Delta \mathrm{FLX} / \Delta \mathrm{EMIS} \\
\left(\mu \mathrm{g} \mathrm{m}^{-2} \mathrm{yr}^{-1} \mathrm{t}^{-1}\right)\end{array}$ \\
\hline CFPP & 48 & 0.07 & 4.11 & 2.38 & 0.10 \\
CFIB & 97 & 0.18 & 10.30 & & 3.02 & 0.25 \\
CEM & 129 & 0.21 & 13.04 & & 2.45 & 0.15 \\
NFMS & 68 & 0.05 & 2.82 & & 0.93 & 0.04 \\
ISP & 32 & 0.05 & 2.74 & & 2.07 & 0.09 \\
\hline
\end{tabular}

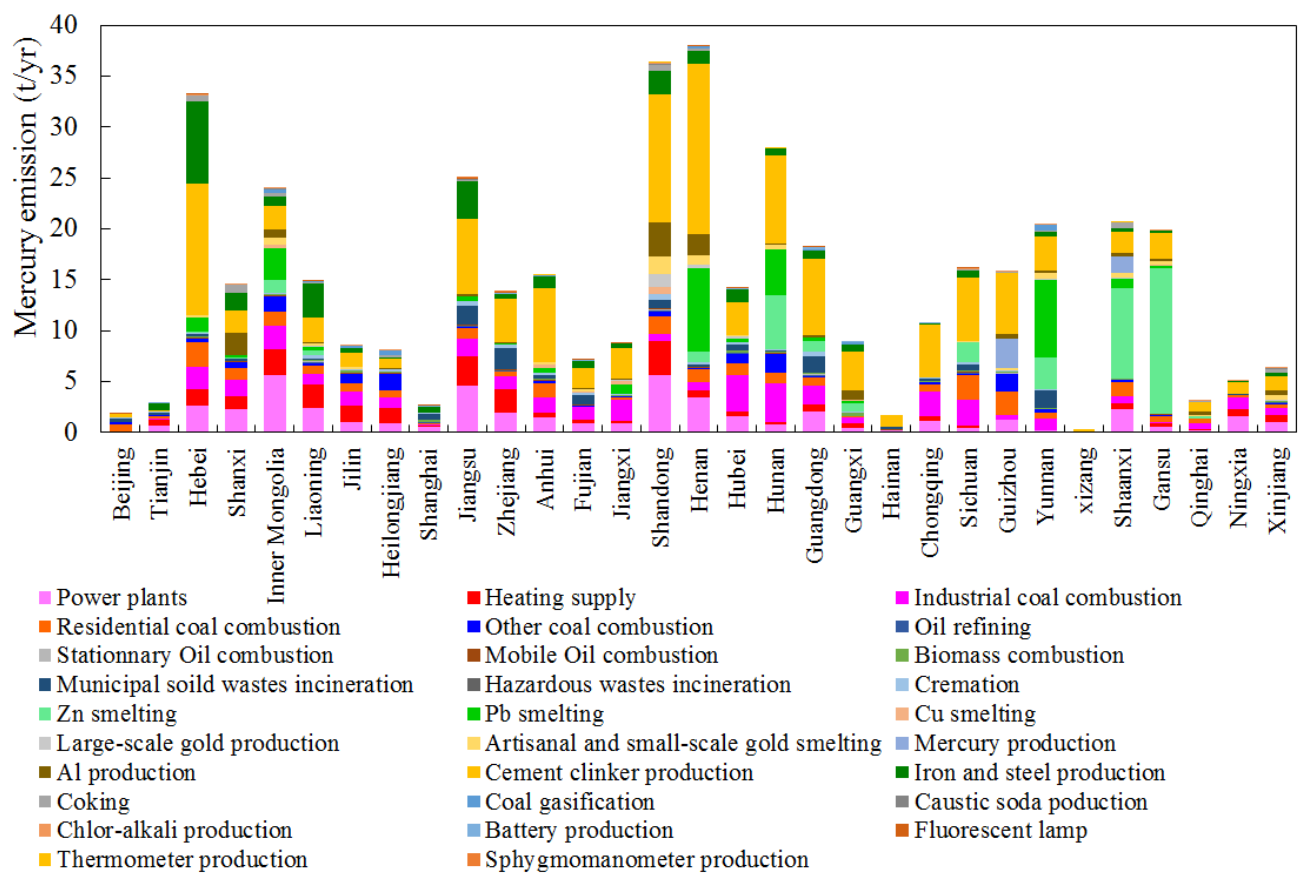

Figure S15. Provincial mercury emission from different sources in 2017 


\section{-References}

(1) Ministry of Ecology and Eenvirenment of the People's Republic of China (MEE). Report for National Mercury Investigation of China; . MEE: Beijing, China, 2012.

(2) China Electricity Council, Chinese statistics of electric power industry. China Power Press: Beijing, China, 2013-2017.

(3) China National Environmental Monitoring Centre (CNEMC). Chinese environmental statistics (collected from provincial environmental protection bureaus). CNEMC: Beijing, China, 2013-2017.

(4) National Energy Statistical Agency of China (NESA). China Energy Statistical Yearbook. NESA: Beijing, China, 2014-2017.

(5) Wu, Q.; Wang, S.; Li, G.; Liang, S.; Lin, C.-J.; Wang, Y.; Cai, S.; Liu, K.; Hao, J. Temporal trend and spatial distribution of speciated atmospheric mercury emissions in China during 1978-2014. Environ. Sci. Technol. 2016, 50 (24), 13428-13435.

(6) Ministry of Ecology and Environment of the People's Republic of China (MEE). Management system of pollutant mission permit (http://permit.mee.gov.cn/).

(7) National Bureau of Statistics of China (NBSC). China Statistical Yearbook. NBSC: Beijing, China, 20142018.

(8) Zhang, L.; Wang, S.; Wang, L.; Wu, Y.; Duan, L.; Wu, Q.; Wang, F.; Yang, M.; Yang, H.; Hao, J.; Liu, X. Updated emission inventories for speciated atmospheric mercury from anthropogenic sources in China. Environ. Sci. Technol. 2015, 49 (5), 3185-3194.

(9) Wu, Y.; Wang, S.; Streets, D. G.; Hao, J.; Chan, M.; Jiang, J. Trends in anthropogenic mercury emissions in China from 1995 to 2003. Environ. Sci. Technol. 2006, 40 (17), 5312-5318.

(10) United Nations Environment Programme (UNEP). Global mercury assessment 2013: sources, emissions, releases and environmental transport. UNEP: Geneva, Switzerland, 2013.

(11) Pacyna, E. G.; Pacyna, J. M.; Sundseth, K.; Munthe, J.; Kindbom, K.; Wilson, S.; Steenhuisen, F.; Maxson, P. Global emission of mercury to the atmosphere from anthropogenic sources in 2005 and projections to 2020. Atmos. Environ. 2010, 44 (20), 2487-2499.

(12) Yang, H. Study on atmospheric mercury emission and control strategies from cement production in China. 
M.S. thesis, Tsinghua University: Beijing, China, 2014.

(13) Zhang, L.; Wang, S.; Meng, Y.; Hao, J. Influence of mercury and chlorine content of coal on mercury emissions from coal-fired power plants in china. Environ. Sci. Technol. 2012, 46 (11), 6385-6392.

(14) Wu, Q. R.; Wang, S. X.; Zhang, L.; Song, J. X.; Yang, H.; Meng, Y. Update of mercury emissions from China's primary zinc, lead and copper smelters, 2000-2010. Atmos. Chem. Phys. 2012, 12 (22), 11153-11163.

(15) Wu, Q.; Gao, W.; Wang, S.; Hao, J. Updated atmospheric speciated mercury emissions from iron and steel production in China during 2000-2015. Atmos. Chem. Phys. 2017, 17 (17), 10423-10433.

(16) Liu, K.; Wang, S.; Wu, Q.; Wang, L.; Ma, Q.; Zhang, L.; Li, G.; Tian, H.; Duan, L.; Hao, J. A highlyresolved mercury emission inventory of Chinese coal-fired power plants. Environ. Sci. Technol. 2018, 52 (4), 2400-2408.

(17) Wang, S.; Luo, K.; Wang, X.; Sun, Y. Estimate of sulfur, arsenic, mercury, fluorine emissions due to spontaneous combustion of coal gangue: An important part of Chinese emission inventories. Environ. Pollut. 2016, 209, 107-113.

(18) Di Natale, F.; Carotenuto, C.; D’Addio, L.; Jaworek, A.; Krupa, A.; Szudyga, M.; Lancia, A. Capture of fine and ultrafine particles in a wet electrostatic scrubber. J. Environ. Chem. Eng. 2015, 3 (1), 349-356.

(19) Wang, X.; You, C. Effects of thermophoresis, vapor, and water film on particle removal of electrostatic precipitator. Aerosol Sci. 2013, 63, 1-9.

(20) Bage Hose Precipiating Committee. Development Report on Bage Hose Precipiating Industry in 2015. 2016, $10,5-20$.

(21) Ministry of Ecology and Eenvirenment of the People's Republic of China (MEE). Emission Standard of Air Pollutants for Thermal Power Plants (GB13223-2011). MEE: Beijing, China, 2011.

(22) National Bureau of Statistics of China (NBSC). China Power Statistical Yearbook. China Power Press: Beijing, China, 2014-2017.

(23) Ministry of Ecology and Environment of the People's Republic of China (MEE). Implementation Plan of Ultra-low Emission and Energy Saving of Coal-fired Power Plant. MEE: Beijing, China, 2015.

(24) Zhang, Y.; Yang, J.; Yu, X.; Sun, P.; Zhao, Y.; Zhang, J.; Chen, G.; Yao, H.; Zheng, C. Migration and emission characteristics of $\mathrm{Hg}$ in coal-fired power plant of China with ultra low emission air pollution control 
devices. Fuel Process. Technol. 2017, 158, 272-280.

(25) Zhao, S.; Duan, Y.; Yao, T.; Liu, M.; Lu, J.; Tan, H.; Wang, X.; Wu, L. Study on the mercury emission and transformation in an ultra-low emission coal-fired power plant. Fuel 2017, 199 (Supplement C), 653-661.

(26) Ministry of Ecology and Eenvirenment of the People's Republic of China (MEE). Emission Standard of Air Pollutants for Cement Industry (GB4915-2013). MEE: Beijing, China, 2013.

(27) Wang, F. Y.; Wang, S. X.; Zhang, L.; Yang, H.; Wu, Q. R.; Hao, J. M. Mercury enrichment and its effects on atmospheric emissions in cement plants of China. Atmos. Environ. 2014, 92, 421-428.

(28) Wu, Q.; Wang, S.; Zhang, L.; Hui, M.; Wang, F.; Hao, J. Flow analysis of the mercury associated with nonferrous ore concentrates: implications on mercury emissions and recovery in China. Environ. Sci. Technol. 2016, 50 (4), 1796-1803.

(29) Wu, Q.; Wang, S.; Hui, M.; Wang, F.; Zhang, L.; Duan, L.; Luo, Y. New Insight into Atmospheric Mercury Emissions from Zinc Smelters Using Mass Flow Analysis. Environ. Sci. Technol. 2015, 49 (6), 3532-3539.

(30) Wang, X.; Lin, C.-J.; Yuan, W.; Sommar, J.; Zhu, W.; Feng, X. Emission-dominated gas exchange of elemental mercury vapor over natural surfaces in China. Atmos. Chem. Phys. 2016, 16 (17), 11125-11143.

(31) Fu, X. W.; Zhang, H.; Yu, B.; Wang, X.; Lin, C. J.; Feng, X. B. Observations of atmospheric mercury in China: a critical review. Atmos. Chem. Phys. 2015, 15 (16), 9455-9476.

(32) Fu, X. W.; Feng, X. B.; Liang, P.; Zhang, H.; Ji, J.; Liu, P. Temporal trend and sources of speciated atmospheric mercury at Waliguan GAW station, Northwestern China. Atmos. Chem. Phys. 2012, 12 (4), 19511964.

(33) Zhang, H.; Fu, X. W.; Lin, C.-J.; Wang, X.; Feng, X. B. Observation and analysis of speciated atmospheric mercury in Shangri-La, Tibetan Plateau, China. Atmos. Chem. Phys. 2015, 15 (2), 9455-9476.

(34) Fu, X. W.; Feng, X. B.; Dong, Z. Q.; Yin, R. S.; Wang, J. X.; Yang, Z. R.; Zhang, H. Atmospheric gaseous elemental mercury (GEM) concentrations and mercury depositions at a high-altitude mountain peak in south China. Atmos. Chem. Phys. 2010, 10 (5), 2425-2437.

(35) Ci, Z.; Zhang, X.; Wang, Z.; Niu, Z. Atmospheric gaseous elemental mercury (GEM) over a coastal/rural site downwind of East China: temporal variation and long-range transport. Atmos. Environ. 2011, 45 (15), 24802487. 
(36) de Foy, B.; Tong, Y.; Yin, X.; Zhang, W.; Kang, S.; Zhang, Q.; Zhang, G.; Wang, X.; Schauer, J. J. First field-based atmospheric observation of the reduction of reactive mercury driven by sunlight. Atmos. Environ. 2016, 134, 27-39.

(37) Zhang, Y.; Liu, R.; Cui, Y.; Zhou, J.; Wang, Y. The characteristic analysis of atmospheric mercury during haze days in Qingdao. Chinese Environ. 2014, 34, 1905-1911.

(38) Liu, M.; Chen, L.; Xie, D.; Sun, J.; He, Q.; Cai, L.; Gao, Z.; Zhang, Y. Monsoon-driven transport of atmospheric mercury to the South China Sea from the Chinese mainland and Southeast Asia-Observation of gaseous elemental mercury at a background station in South China. Environ. Sci. Pollut. Res. 2016, 23 (21), 21631-21640.

(39) Fu, X.; Zhu, W.; Zhang, H.; Sommar, J.; Yu, B.; Yang, X.; Wang, X.; Lin, C.-J.; Feng, X. Depletion of atmospheric gaseous elemental mercury by plant uptake at Mt. Changbai, Northeast China. Atmos. Chem. Phys. 2016, $16(20), 12861-12873$.

(40) Zhang, H.; Fu, X.; Lin, C.-J.; Shang, L.; Zhang, Y.; Feng, X.; Lin, C. Monsoon-facilitated characteristics and transport of atmospheric mercury at a high-altitude background site in southwestern China. Atmos. Chem. Phys. 2016, 16 (20), 13131-13148.

(41) Fu, X.; Yang, X.; Lang, X.; Zhou, J.; Zhang, H.; Yu, B.; Yan, H.; Lin, C. J.; Feng, X. Atmospheric wet and litterfall mercury deposition at urban and rural sites in China. Atmos. Chem. Phys. 2016, 16 (18), 11547-11562. (42) Lang, X. Mercury in atmospheric precipitation and litterfall in Mt.Ailao and Mt.Damei. Master's dissertation. M.S. thesis, Guizhou University: Guiyang, China, 2014.

(43) Huang, J.; Kang, S.; Zhang, Q.; Yan, H.; Guo, J.; Jenkins, M. G.; Zhang, G.; Wang, K. Wet deposition of mercury at a remote site in the Tibetan Plateau: Concentrations, speciation, and fluxes. Atmos. Environ. 2012, $62,540-550$.

(44) Fu, X.; Feng, X.; Zhu, W.; Zheng, W.; Wang, S.; Lu, J. Y. Total particulate and reactive gaseous mercury in ambient air on the eastern slope of the Mt. Gongga area, China. Appl. Geochem. 2008, 23 (3), 408-418.

(45) Fu, X.; Feng, X.; Zhu, W.; Rothenberg, S.; Yao, H.; Zhang, H. Elevated atmospheric deposition and dynamics of mercury in a remote upland forest of southwestern China. Environ. Pollut. 2010, 158 (6), 23242333. 
(46) Wan, Q.; Feng, X.; Lu, J.; Zheng, W.; Song, X.; Li, P.; Han, S.; Xu, H. Atmospheric mercury in Changbai Mountain area, northeastern China II. The distribution of reactive gaseous mercury and particulate mercury and mercury deposition fluxes. Environ. Res. 2009, 109 (6), 721-727.

(47) Guo, Y.; Feng, X.; Li, Z.; He, T.; Yan, H.; Meng, B.; Zhang, J.; Qiu, G. Distribution and wet deposition fluxes of total and methyl mercury in Wujiang River Basin, Guizhou, China. Atmo. Environ. 2008, 42 (30), 70967103. 